\title{
The pendulum-slosh problem: simulation using a time-dependent conformal mapping
}

\author{
by M.R. Turner ${ }^{1}$, T.J. Bridges \& H. Alemi Ardakani \\ Department of Mathematics, University of Surrey, \\ Guildford, Surrey GU2 7XH, England
}

- Abstract -

Suspending a rectangular vessel which is partially filled with fluid from a single rigid pivoting pole produces an interesting theoretical model with which to investigate the dynamic coupling between fluid motion and vessel rotation. The exact equations for this coupled system are derived with the fluid motion governed by the Euler equations relative to the moving frame of the vessel, and the vessel motion governed by a modified forced pendulum equation. The nonlinear equations of motion for the fluid are solved numerically via a timedependent conformal mapping, which maps the physical domain to a rectangle in the computational domain with a time dependent conformal modulus. The numerical scheme expresses the implicit free-surface boundary conditions as two explicit partial differential equations which are then solved via a pseudospectral method in space. The coupled system is integrated in time with a fourth-order Runge-Kutta method. The starting point for the simulations is the linear neutral stability contour discovered by Turner, Alemi Ardakani \& Bridges (2014, J. Fluid Struct. 52, 166-180). Near the contour the nonlinear results confirm the instability boundary, and far from the neutral curve (parameterised by longer pole lengths) nonlinearity is found to significantly alter the vessel response. Results are also presented for an initial condition given by a superposition of two sloshing modes with approximately the same frequency from the linear characteristic equation. In this case the fluid initial conditions generate large nonlinear vessel motions, which may have implications for systems designed to oscillate in a confined space or on the slosh-induced-rolling of a ship.

\footnotetext{
${ }^{1}$ Corresponding Author: m.turner@surrey.ac.uk
} 


\section{Introduction}

The pendulum-slosh problem consists of a vessel, partially filled with fluid, rigidly attached to a pivot point in the plane, with coupling between the fluid motion and rotation of the vessel about the pivot point. This problem is the simplest configuration which couples vessel rotation and fluid motion, and provides a simplified model for the slosh-induced rolling of a ship as shown schematically in Figure 1. An example of a roll destabilization due to sloshing occurs on Alaskan king crab boats which can capsize when water trapped on the deck sloshes from side to side creating unintended moments enhancing the roll motion of the boat (Dillingham, 1981; Caglayan and Storch, 1982; Adee and Caglayan, 1982).

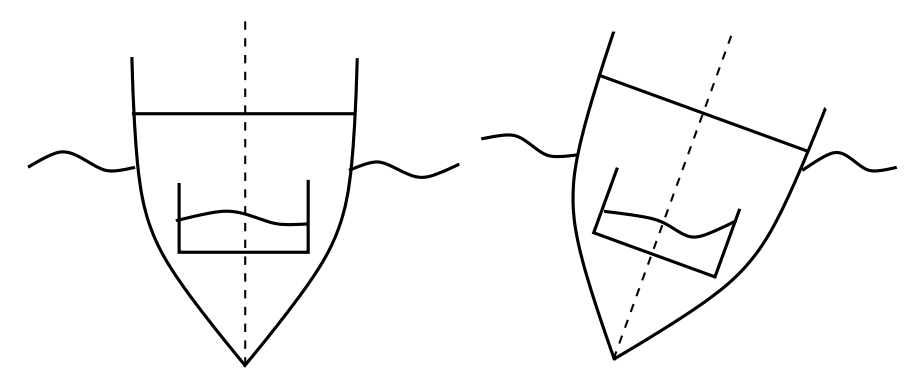

Figure 1: Interaction between fluid sloshing and ship roll motion.

The pendulum-slosh problem was one of the first examples of slosh-vessel interaction to be studied (Moiseev, 1953; Abramson et al., 1961; Moiseyev and Rumyantsev, 1968). Moiseyev and Rumyantsev (1968) derived the governing linear equations, and consequently the characteristic equation, by considering the added mass coefficients for the fluid and a Lagrangian construction for the vessel. The pendulum-slosh problem is more difficult, due to the rotary motion of the vessel, than coupling with horizontal motion, such as tuned liquid damping (TLD) systems (e.g. Frandsen (2005); Xue et al. (2000); Idir et al. (2009); Gardarsson et al. (2001); Alemi Ardakani and Bridges (2010)). The motion of a fluid in a stationary or forced vessel, whether studied experimentally, theoretically or numerically, is already very complicated. The works by Moiseyev and Rumyantsev (1968), Ibrahim (2005) and Faltinsen and Timokha (2009), and the references therein, highlight the problems in these areas. The problem of coupled dynamics, particularly with vessel rotation, adds an additional layer of complexity to this already difficult problem because it can happen that the waves are excited or calmed by the vessel's motion, or the vessel's motion may be excited or calmed by the motion of the waves.

The starting point for this paper is the linear analysis of the pendulum-slosh problem of Turner et al. (2015). They studied the linear system for a vessel with rectangular crosssection in depth and discovered an instability when the pivot point $l=\hat{l}+d$ satisfies

$$
(1+R) l<\frac{1}{2} h_{0}+\frac{1}{12} \frac{L^{2}}{h_{0}}
$$

where

$$
R=\frac{m_{v}}{m_{f}}
$$


is the ratio of the dry vessel mass $m_{v}$ and the fluid mass $m_{f}$. Here $L$ is the vessel length, $d$ its height, $\hat{l}$ is the rigid pole length and $h_{0}$ is the mean fluid height as shown in the schematic diagram in figure 2. A remarkable feature of the above instability is that $l$ can be larger than $h_{0}$, i.e. above the height of the quiescent fluid, depending on the parameter values of the system. the configuration in figure 2 is free to rotate in the vertical plane such that the rigid pole makes an angle $\theta$ with the downward vertical, and the vessel is partially filled with an inviscid, incompressible fluid of mass $m_{f}$ and constant density $\rho$,

$$
m_{f}=\int_{0}^{L} \rho h(x, t) d x, \quad \text { with } \quad \frac{d}{d t} m_{f}=0 .
$$

Here $y=h(x, t)$ is the free surface of the fluid and $\mathbf{x}=(x, y)$ is a coordinate system fixed to the moving vessel with the origin at the bottom left-hand corner of the vessel, and we also define $\widehat{\mathbf{X}}=(\widehat{X}, \widehat{Y})$ to be a planar fixed coordinate system with the origin at the point at which the vessel pivots.

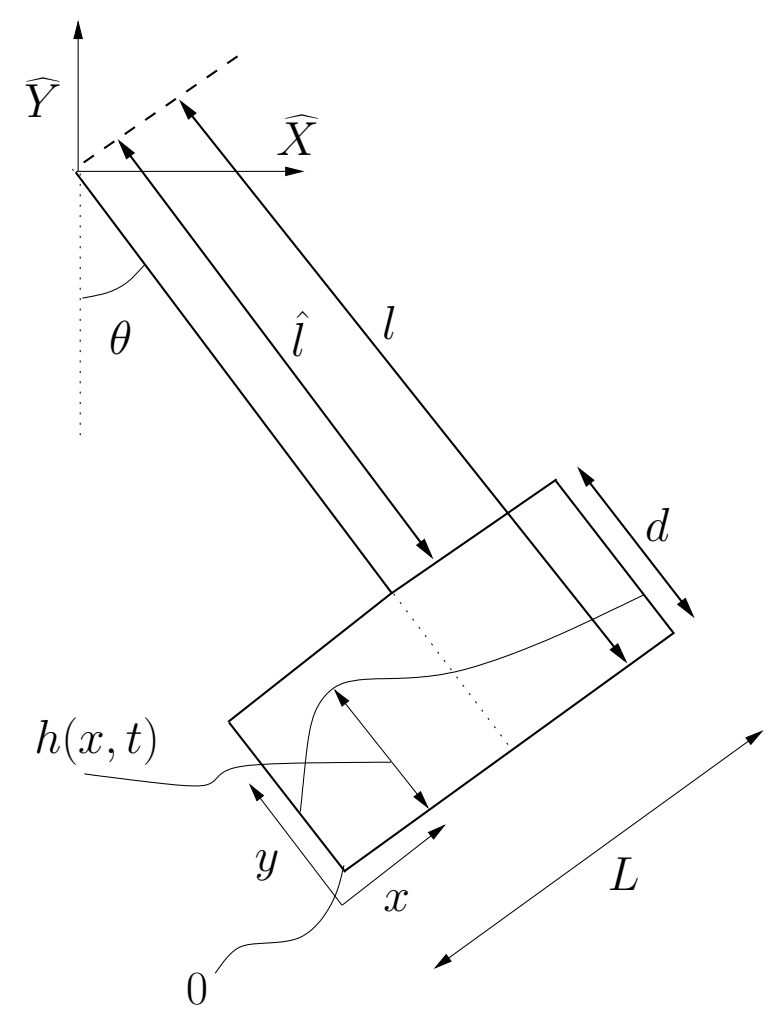

Figure 2: Schematic of the pendulum-slosh problem.

In this paper we present numerical simulations of the fully nonlinear two-dimensional equations for a variety of parameter values both close to, and away from the above linear neutral stability contour. There has been much work on numerical solutions to both free and forced sloshing problems using techniques such as finite difference, pseudo-spectral, Lagrangian-Eulerian boundary fitted grids, finite elements and domain mappings (e.g. Telste, 1985; Ferrant and Le Touze, 2001; Ushijima, 1998; Hirt and Nichols, 1981; Frandsen, 2004). The main problem with numerical simulations is how to treat the unknown position of the free-surface. Numerical models tend to use either a Lagrangian method to track 
the free-surface or mapping to a simpler domain. The disadvantage of the first approach is that surface velocities are difficult to predict correctly, hence free-surface smoothing is often used which introduces errors into the solution. The use of mappings overcomes this problem, but they cannot easily predict features such as wetting and drying of the vessel bottom. In shallow water, the Lagrangian particle path approach is more fruitful, and Alemi Ardakani and Bridges (2010) constructed a fast-numerical algorithm based on a symplectic integrator to simulate coupled TLD motion. However, this approach can not be easily extended to finite depth fluids. To solve the two-dimensional nonlinear coupled system in this paper we present a method based upon a time-dependent conformal mapping, in which the volume of the fluid bounded by the vessel and the unknown freesurface in physical space, is mapped to a rectangle with a time-dependent aspect ratio in the mapped computational domain.

The idea of time-dependent conformal mappings for water wave simulations goes back to Byatt-Smith (1971) and Whitney (1971), but the approach we use is based upon that of Dyachenko et al. (1996) who used the Hilbert transform to relate the conjugate harmonic functions along the free surface and then introduced a fast numerical algorithm based upon pseudo-spectral methods for spatially periodic flows. This efficient numerical algorithm is possible because Dyachenko et al. (1996) formulated the implicit kinematic and dynamic boundary conditions on the free surface into two explicit partial differential equations for the free surface evolution. This theory was originally developed for infinite depth flows, but has since been extended to finite depth flows (Dyachenko et al., 1999; Choi and Camassa, 1999; Li et al., 2004; Viotti et al., 2013), and a review article following up on this approach with extensive references was published by Shamin (2009). The method for the finite depth case centres around defining a time-dependent conformal mapping which maps the boundary of the physical fluid domain to a rectangle in the mapped domain with a time-dependent aspect ratio known as the conformal modulus, which has to be found as part of the solution (Challis and Burley, 1982; Seidl and Klose, 1985; Papamichael and Stylianopoulos, 2010).

Curiously, previous studies do not emphasize the significance of the conformal modulus which is time-dependent and has to be solved for as part of the solution. The conformal modulus is a purely geometric requirement of the mapping and does not appear to have any physical significance. Hence there is a temptation to find a strategy that allows fixing the conformal modulus at a chosen value. When the flow is steady the mapping is fixed and the conformal modulus is fixed. In this case, if the specific value of the mean depth is not important, the value of the conformal modulus can be fixed, and then the value of the depth determined as part of the solution. This strategy is used in Vanden-Broeck and Schwartz (1979) and Constantin et al. (2014). However, this strategy fails in the timedependent case since fixing the conformal modulus will lead to a time-dependent value of the depth! The work of Turner and Bridges (2015) showed both analytically and numerically that the explicit time-dependent form of the conformal modulus is vital in order to correctly determine the evolution of the free-surface, especially in nonlinear interactions. They also presented a numerical scheme which generalises that of Dyachenko et al. (1996) by introducing the "Garrick-Hilbert transform", which is the required generalisation of the Hilbert transform to doubly-connected regions, as well as a numerical scheme for the conformal modulus. In this paper we derive the corresponding explicit free-surface PDEs for the pendulum-slosh problem and solve them along with a non-linear pendulum equation for the vessel motion using a generalisation to the coupled system of the approach 
presented in Turner and Bridges (2015).

Nonlinear results are presented for non-breaking waves on either side of the linear neutral stability contour in parameter space. Nonlinear results are also presented as the pole length is varied (for a fixed fluid mass) highlighting the vessel behaviour far from the neutral contour. In these simulations the initial conditions for the fluid are given by the linear form of the fundamental linear sloshing mode. These results are then compared to the vessel motion obtained for experimentally realistic initial conditions in order to contrast differences in behaviour of the dynamic feedback. Finally nonlinear simulations are given for a parameter set containing the superposition of two linear anti-symmetric sloshing modes with similar frequencies. Here the nonlinearity produces a vessel motion with an oscillation amplitude much larger than the corresponding linear result. This result may have implications for a physical system designed to oscillate in a confined space or on the slosh-induced-rolling of a ship.

The structure of the paper is as follows. The formulation of the governing nonlinear equations for the coupled pendulum problem are given in $\S 2$. In $\S 3$ we derive the nonlinear PDEs governing the evolution of the free-surface, formulate the time-dependent conformal mapping and present the numerical scheme. The results of the nonlinear simulations are given in $\S 4$ and conclusions and further discussions can be found in $\S 5$.

\section{Formulation of governing equations}

\section{$2.1 \quad$ Formulation}

A schematic of the physical problem addressed in this paper is given in figure 2. The two Cartesian coordinate systems are related via

$$
\widehat{\mathbf{X}}=\widehat{\mathbf{Q}}(t)(\mathbf{x}+\mathbf{d}), \quad \text { where } \quad \widehat{\mathbf{Q}}(t)=\left(\begin{array}{cc}
\cos \theta & -\sin \theta \\
\sin \theta & \cos \theta
\end{array}\right)
$$

is a rotation tensor in $\mathbb{R}^{2}$ and $\mathbf{d}=\left(d_{1} d_{2}\right)^{T}=(-L / 2-l)^{T}$, is the displacement of the axis of rotation from the origin of the body frame. We have assumed for simplicity that the rigid pole is fixed along the centre line of the vessel (i.e. $d_{1}=-L / 2$ ), but we leave the quantities $d_{1}$ and $d_{2}$ in the analysis to simplify the algebra.

We define the velocity vector $\mathbf{u}=(u, v)$ to be the Eulerian velocity vector in the body coordinate system and the Euler equations of motion in this frame are

$$
\begin{aligned}
& \frac{D u}{D t}+\frac{1}{\rho} \frac{\partial p}{\partial x}=-g \sin \theta+2 \dot{\theta} v+\ddot{\theta}\left(y+d_{2}\right)+\dot{\theta}^{2}\left(x+d_{1}\right), \\
& \frac{D v}{D t}+\frac{1}{\rho} \frac{\partial p}{\partial y}=-g \cos \theta-2 \dot{\theta} u-\ddot{\theta}\left(x+d_{1}\right)+\dot{\theta}^{2}\left(y+d_{2}\right),
\end{aligned}
$$

where $g$ denotes the constant acceleration due to gravity, which acts in the negative $\widehat{Y}$ direction and the dots denote differentiation with respect to $t$ (Turner et al., 2015).

The fluid is incompressible, so it satisfies the continuity equation $u_{x}+v_{y}=0$, while the boundary conditions are the no penetration conditions on the rigid vessel walls, and 
the dynamic and kinematic conditions on the free-surface $y=h(x, t)$,

$$
\begin{aligned}
v=0 & \text { on } \quad y=0, \\
u=0 & \text { on } \quad x=0, L, \\
p=0 & \text { on } \quad y=h(x, t), \\
h_{t}+u h_{x}=v & \text { on } \quad y=h(x, t) .
\end{aligned}
$$

The fluid motion within the vessel is dynamically coupled to the motion of the vessel via a pendulum-like equation which is derived by starting with a Lagrangian for the vessel motion,

$$
\mathcal{L}=\int_{t_{1}}^{t_{2}} \mathscr{L} d t
$$

where

$$
\begin{aligned}
\mathscr{L}= & \int_{0}^{L} \int_{0}^{h}\left[\frac{1}{2}\left(u^{2}+v^{2}\right)-\dot{\theta} u\left(y+d_{2}\right)+\dot{\theta} v\left(x+d_{1}\right)+\frac{1}{2} \dot{\theta}^{2}\left(\left(x+d_{1}\right)^{2}+\left(y+d_{2}\right)^{2}\right)\right. \\
& \left.-g\left(\sin \theta\left(x+d_{1}\right)+\cos \theta\left(y+d_{2}\right)\right)\right] \rho d y d x+\frac{1}{2} m_{v}\left[\left(x_{v}+d_{1}\right)^{2}+\left(y_{v}+d_{2}\right)^{2}\right] \dot{\theta}^{2} \\
& -m_{v} g\left(\left(x_{v}+d_{1}\right) \sin \theta+\left(y_{v}+d_{2}\right) \cos \theta\right) .
\end{aligned}
$$

(Alemi Ardakani, 2010; Turner et al., 2015). Here $\left(x_{v}, y_{v}\right)$ is the position of the centre of mass of the vessel in the body frame coordinates. The vessel differential equation is then derived via the Euler-Lagrange equation, $\frac{d}{d t}\left(\frac{\partial \mathscr{L}}{\partial \dot{\theta}}\right)-\frac{\partial \mathscr{L}}{\partial \theta}=0$, for $\theta$

$$
\begin{aligned}
& \left(m_{v}\left[\left(x_{v}+d_{1}\right)^{2}+\left(y_{v}+d_{2}\right)^{2}\right]+\int_{0}^{L} \int_{0}^{h}\left[\left(x+d_{1}\right)^{2}+\left(y+d_{2}\right)^{2}\right] \rho d y d x\right) \ddot{\theta} \\
& +\dot{\theta} \frac{d}{d t} \int_{0}^{L} \int_{0}^{h}\left[\left(x+d_{1}\right)^{2}+\left(y+d_{2}\right)^{2}\right] \rho d y d x+m_{v} g\left[\left(x_{v}+d_{1}\right) \cos \theta-\left(y_{v}+d_{2}\right) \sin \theta\right] \\
& +g \int_{0}^{L} \int_{0}^{h}\left[\cos \theta\left(x+d_{1}\right)-\sin \theta\left(y+d_{2}\right)\right] \rho d y d x=\frac{d}{d t}\left[\int_{0}^{L} \int_{0}^{h}\left[u\left(y+d_{2}\right)-v\left(x+d_{1}\right)\right] \rho d y d x\right](2.8)
\end{aligned}
$$

The fluid flow is not irrotational, due to the vessel rotation. This rotationality is in contrast to the TLD or the Cooker bi-pendulum experiment (Cooker, 1994; Frandsen, 2005; Alemi Ardakani et al., 2012) where the base of the vessel remains horizontal during motion. However, the rotationality can be treated by splitting the flow field into a rotational part and an irrotational part and introducing a velocity potential $\phi(x, y, t)$ for the irrotational part. Thus we set

$$
u=\frac{\partial \phi}{\partial x}, \quad \text { and } \quad v=\frac{\partial \phi}{\partial y}-2 \dot{\theta}\left(x+d_{1}\right)
$$

(see Turner et al. (2015) for background on this particular form for the splitting). The continuity equation then leads to Laplace's equation for $\phi$

$$
\phi_{x x}+\phi_{y y}=0 \quad \text { in } \quad 0 \leq y \leq h(x, t), \quad 0 \leq x \leq L,
$$

and integrating (2.1) and (2.2) with respect to $x$ and $y$ respectively leads to Bernoulli's equation for the pressure

$$
\begin{aligned}
& \frac{p}{\rho}+\phi_{t}+\frac{1}{2}\left(\phi_{x}^{2}+\phi_{y}^{2}\right)-2 \dot{\theta}\left(x+d_{1}\right) \phi_{y}-\ddot{\theta}\left(y+d_{2}\right)\left(x+d_{1}\right) \\
& -\frac{1}{2} \dot{\theta}^{2}\left[-3\left(x+d_{1}\right)^{2}+\left(y+d_{2}\right)^{2}\right]+g(x \sin \theta+y \cos \theta)=B e(t),
\end{aligned}
$$


where $B e(t)$ is the Bernoulli function which, without loss of generality, can be absorbed into $\phi(x, y, t)$. Therefore, the dynamic free-surface boundary condition (2.5) becomes

$$
\begin{aligned}
& \phi_{t}+\frac{1}{2}\left(\phi_{x}^{2}+\phi_{y}^{2}\right)-2 \dot{\theta}\left(x+d_{1}\right) \phi_{y}-\ddot{\theta}\left(h+d_{2}\right)\left(x+d_{1}\right) \\
& -\frac{1}{2} \dot{\theta}^{2}\left[-3\left(x+d_{1}\right)^{2}+\left(h+d_{2}\right)^{2}\right]+g(x \sin \theta+h \cos \theta)=0, \quad \text { on } y=h(x, t) .
\end{aligned}
$$

In terms of the velocity potential $\phi(x, y, t)$, the other boundary conditions are

$$
\begin{aligned}
& \frac{\partial \phi}{\partial x}=0, \quad \text { on } \quad x=0, L, \\
& \frac{\partial \phi}{\partial y}=2 \dot{\theta}\left(x+d_{1}\right), \quad \text { on } \quad y=0,
\end{aligned}
$$

and the kinematic free-surface boundary condition becomes

$$
h_{t}+\phi_{x} h_{x}=\phi_{y}-2 \dot{\theta}\left(x+d_{1}\right) \quad \text { on } \quad y=h(x, t) .
$$

Substituting the velocity potential into (2.8) gives the form of the vessel equation as

$$
\begin{aligned}
& \left(m_{v}\left[\left(x_{v}+d_{1}\right)^{2}+\left(y_{v}+d_{2}\right)^{2}\right]+\int_{0}^{L} \int_{0}^{h}\left[\left(x+d_{1}\right)^{2}+\left(y+d_{2}\right)^{2}\right] \rho d y d x\right) \ddot{\theta} \\
& +\dot{\theta} \frac{d}{d t} \int_{0}^{L} \int_{0}^{h}\left[\left(x+d_{1}\right)^{2}+\left(y+d_{2}\right)^{2}\right] \rho d y d x+m_{v} g\left(\left(x_{v}+d_{1}\right) \cos \theta-\left(y_{v}+d_{2}\right) \sin \theta\right) \\
& +g \int_{0}^{L} \int_{0}^{h}\left[\cos \theta\left(x+d_{1}\right)-\sin \theta\left(y+d_{2}\right)\right] \rho d y d x \\
& =\frac{d}{d t}\left[\int_{0}^{L} \int_{0}^{h}\left[\phi_{x}\left(y+d_{2}\right)-\left(\phi_{y}-2 \dot{\theta}\left(x+d_{1}\right)\right)\left(x+d_{1}\right)\right] \rho d y d x\right] .
\end{aligned}
$$

Although the equation for the vessel looks quite complicated, it is a nonlinear pendulum equation with coefficients and forcing that depend on the fluid motion.

\subsection{Linear solutions and natural frequencies}

In the results section, $\S 4$, we utilize results from the linear theory as initial conditions, as well as for determining the natural frequencies of the system. Hence we briefly summarize the key results on the linear problem from Turner et al. (2015).

The characteristic equation for this system is found by considering linear solutions of (2.10)-(2.15), about a state of quiescent fluid where $\phi=-g h_{0} t, h=h_{0}, \theta=0$ and $h_{0}$ is a constant, of the form

$$
\begin{aligned}
\phi(x, y, t) & =-g h_{0} t-\frac{1}{2}\left[\mathrm{i} \widehat{\phi}(x, y) e^{\mathrm{i} \omega t}+\mathrm{c.c}\right] \\
h(x, t) & =h_{0}+\frac{1}{2}\left[\widehat{h}(x) e^{\mathrm{i} \omega t}+\text { c.c }\right] \\
\theta(t) & =\frac{1}{2} \widehat{\theta} e^{\mathrm{i} \omega t}+\text { c.c }
\end{aligned}
$$

where $\omega=\Omega_{r}+i \Omega_{i}$ is the complex angular frequency, $i=\sqrt{-1}$, c.c denotes the complex conjugate and $\widehat{\phi}$ and $\widehat{h}$ are complex functions with $\widehat{\theta}$ real, such that $|\widehat{\phi}|,|\widehat{h}|,|\widehat{\theta}| \ll 1$. The 
exact forms of $\widehat{\phi}$ and $\widehat{h}$, as well as a complete derivation of the characteristic equation, are given in Turner et al. (2015), except here we have multiplied the linear perturbations by $-\mathrm{i}$. Also, for definiteness, we fix the position of the centre of mass of the vessel. To simplify the algebra, we set the centre of mass of the dry vessel to be at $\left(x_{v}, y_{v}\right)=\left(-d_{1}, 0\right)$, i.e. at the centre of the base of the vessel. Then Turner et al. (2015) show that the nondimensional characteristic equation takes the form

$$
\Delta(s)=P(s) D(s)=D(s) \prod_{n=1}^{\infty} \widehat{\sigma}_{n}
$$

where

$$
\begin{aligned}
D(s)= & {\left[G-\frac{2 \delta^{2} G^{2}}{(1+R)^{2}}-\frac{2 G^{2}}{3(1+R)^{2}}-s^{2}\left(1+R-\frac{4 \delta^{2} G^{2}}{3(1+R)^{2}}-\frac{4 \delta^{2} G}{1+R}+\frac{16 \delta^{4} G^{2}}{3(1+R)^{2}}\right)\right.} \\
& +32 \sum_{n=0}^{\infty} \frac{1}{\gamma_{n}^{3} \sigma_{n}}\left[T_{n}\left(s^{4}\left(\frac{4 \delta^{2} G}{1+R}-1\right)^{2}-\frac{G^{2}}{(1+R)^{2}}\right)-\frac{8 \delta s^{4} G}{\gamma_{n}(1+R)}\left(\frac{4 \delta^{2} G}{1+R}-1+\frac{8 \delta T_{n} G}{\gamma_{n}(1+R)}\right)\right. \\
& \left.\left.+\frac{2 s^{2} \sigma_{n} G}{\gamma_{n}(1+R)}+\frac{8 \delta s^{2} G}{C_{n} \gamma_{n}(1+R)}\left(\frac{G}{1+R}\left(3 C_{n}-2\right)+2 s^{2}\left(\frac{4 \delta^{2} G}{1+R}-1\right)\right)\right]\right] \prod_{n=0}^{\infty} \sigma_{n} .
\end{aligned}
$$

The quantities can be expressed in terms of dimensional quantities via

$$
R=\frac{m_{v}}{m_{f}}, \quad G=\frac{L^{2}\left(m_{v}+m_{f}\right)}{4 h_{0} m_{f} l}=\frac{L\left(m_{v}+m_{f}\right)}{4 \delta m_{f} l}, \quad \delta=\frac{h_{0}}{L}, \quad \frac{l}{L}=\frac{(1+R)}{4 \delta G}, \quad s=\frac{\omega L}{2 \sqrt{g h_{0}}},
$$

where the $n$-dependent parameters are

$$
\begin{aligned}
& \gamma_{n}=\alpha_{n} L=(2 n+1) \pi, \quad \widehat{\gamma}_{n}=\beta_{n} L=2 n \pi, \\
& \sigma_{n}=\frac{L}{g}\left(\omega^{2}-g \alpha_{n} T_{n}\right)=4 \delta s^{2}-\gamma_{n} T_{n}, \quad \widehat{\sigma}_{n}=4 \delta s^{2}-\widehat{\gamma}_{n} \widehat{T}_{n}, \\
& T_{n}=\tanh \left(\gamma_{n} \delta\right), \quad \widehat{T}_{n}=\tanh \left(\widehat{\gamma_{n}} \delta\right), \quad C_{n}=\cosh \left(\gamma_{n} \delta\right) .
\end{aligned}
$$

The characteristic equation is solved numerically for the complex non-dimensional frequency $s=S_{r}+\mathrm{i} S_{i}$. Before presenting simulations of the nonlinear equations we first formulate the time-dependent conformal mapping technique.

\section{Time-dependent conformal mapping formulation}

In order to use periodicity, we first extend the problem to $x \in[0,2 L]$ by defining the even extension of $\phi(x, y, t)$ as

$$
\phi(x, y, t)=\phi(2 L-x, y, t) \quad \text { for } \quad L \leq x \leq 2 L .
$$

This ensures that all of the modes for $x \in[0, L]$, both symmetric and anti-symmetric, are periodic for $x \in[0,2 L]$ and thus we can utilize Fourier analysis in combination with the conformal mappings (Dyachenko et al., 1996; Bridges and Donaldson, 2011; Turner and Bridges, 2015).

The conformal mapping takes the physical domain $x \in[0,2 L], y \in[0, h(x, t)]$ to the computational domain $\mu \in[0,2 L]$ and $\nu=[-Q(t), 0]$ where $Q(t)$ is the time dependent 

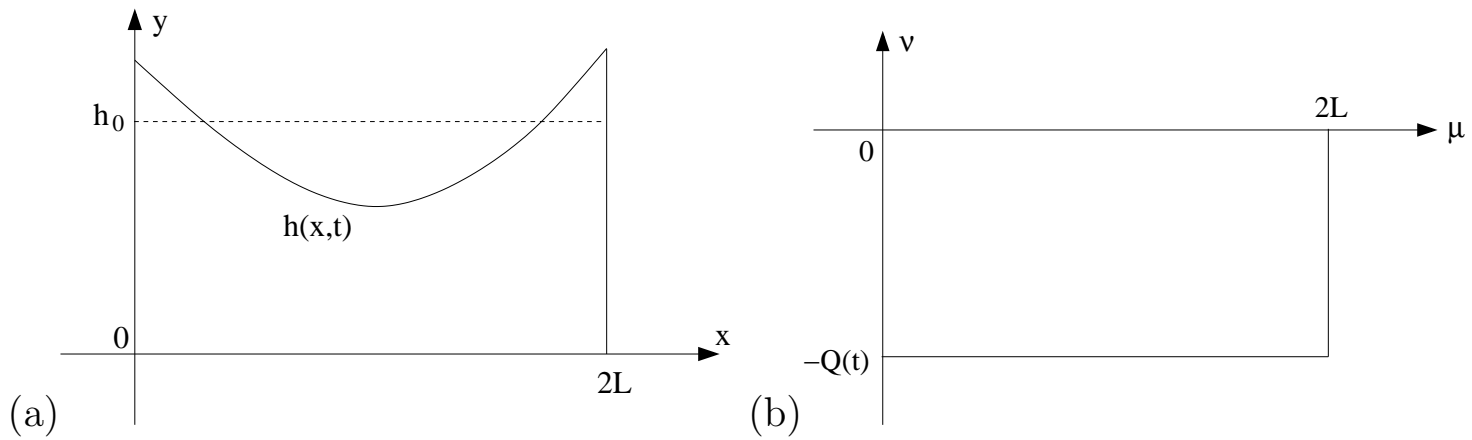

Figure 3: Schematic of (a) the physical domain which is mapped into (b) a fixed rectangular computational domain by the time-dependent conformal mapping. The timedependent aspect ratio of the rectangle, $Q(t)$, is known as the conformal modulus.

conformal modulus which needs to be found as part of the solution procedure. A schematic of the physical and computational domains are shown in figure 3.

In the computational domain the physical coordinates are $x=x(\mu, \nu, t)$ and $y=$ $y(\mu, \nu, t)$. On $\nu=0$ in the computational domain these coordinates define a parametric form of the free surface

$$
(X(\mu, t), Y(\mu, t))=(x(\mu, 0, t), y(\mu, 0, t)) \quad \text { for } \quad \mu=[0,2 L] .
$$

It is assumed throughout this paper that the surface is non-degenerate everywhere, that is $J=X_{\mu}^{2}+Y_{\mu}^{2} \neq 0$ for all $t$ and $0 \leq \mu \leq 2 L$.

Both the coordinates, $x(\mu, \nu, t)+\mathrm{i} y(\mu, \nu, t)$, and the complex potential, $\phi(\mu, \nu, t)+$ $\mathrm{i} \psi(\mu, \nu, t)$, are time-dependent holomorphic functions, satisfying the Cauchy-Riemann equations,

$$
x_{\mu}=y_{\nu}, \quad x_{\nu}=-y_{\mu}, \quad \phi_{\mu}=\psi_{\nu}, \quad \phi_{\nu}=-\psi_{\mu} .
$$

Here $\psi$, the stream function, and $\phi$, the velocity potential, satisfy Laplace's equation in the computational domain. It remains to transform the boundary condition from the physical domain to the computational domain. Following the approach of Bridges and Donaldson (2011) (cf. equations (A-7) and (3.8) in Bridges and Donaldson (2011)), with the details confined to appendix A, the boundary conditions become

$$
\begin{array}{rll}
\frac{1}{J}\left(\phi_{\mu} x_{\mu}+\psi_{\mu} y_{\mu}\right)=0 & \text { on } & \mu=0,2 L, \\
x_{\mu} \psi_{\mu}=-2 \dot{\theta} J\left(x+d_{1}\right) & \text { on } & \nu=-Q(t), \\
X_{\mu} Y_{t}-Y_{\mu} X_{t}=-\Psi_{\mu}-2 \dot{\theta} X_{\mu}\left(X+d_{1}\right) & \text { on } & \nu=0, \\
J \Phi_{t}-\left(Y_{\mu} Y_{t}+X_{\mu} X_{t}\right) \Phi_{\mu}-\left(Y_{\mu} X_{t}-X_{\mu} Y_{t}\right) \Psi_{\mu}+\frac{1}{2}\left(\Phi_{\mu}^{2}+\Psi_{\mu}^{2}\right) & \\
-2 \dot{\theta}\left(X+d_{1}\right)\left(Y_{\mu} \Phi_{\mu}-X_{\mu} \Psi_{\mu}\right)-\ddot{\theta} J\left(Y+d_{2}\right)\left(X+d_{1}\right) & \\
-\frac{J}{2} \dot{\theta}^{2}\left[-3\left(X+d_{1}\right)^{2}+\left(Y+d_{2}\right)^{2}\right]+g J(X \sin \theta+Y \cos \theta)=0 & \text { on } \quad \nu=0,
\end{array}
$$

where

$$
\Phi(\mu, t)+\mathrm{i} \Psi(\mu, t)=\phi(\mu, 0, t)+\mathrm{i} \psi(\mu, 0, t),
$$

is the complex potential evaluated on the free surface. The boundary conditions (3.2)(3.5) are valid for $\mu \in[0, L]$, with a similar set with $x$ replaced by $2 L-x$ valid for $x \in[L, 2 L]$, completing the boundary value problem. 


\subsection{Determining the conjugate function transformations}

The most general solutions for $x(\mu, \nu, t), y(\mu, \nu, t), \phi(\mu, \nu, t)$ and $\psi(\mu, \nu, t)$ which satisfy the side wall boundary conditions (3.2), give $x=0, L, 2 L$ at $\mu=0, L, 2 L$, and satisfy the Cauchy-Riemann equations (3.1), are

$$
\begin{aligned}
y(\mu, \nu, t) & =h_{0}+B_{0}(t)+\nu+\sum_{n=1}^{\infty}\left(B_{n}(t) \cosh \frac{n \pi}{L} \nu+A_{n}(t) \sinh \frac{n \pi}{L} \nu\right) \cos \frac{n \pi}{L} \mu \\
x(\mu, \nu, t) & =\mu+\sum_{n=1}^{\infty}\left(A_{n}(t) \cosh \frac{n \pi}{L} \nu+B_{n}(t) \sinh \frac{n \pi}{L} \nu\right) \sin \frac{n \pi}{L} \mu \\
\phi(\mu, \nu, t) & =C_{0}(t)+C_{01}(t) \nu+\sum_{n=1}^{\infty}\left(C_{n}(t) \cosh \frac{n \pi}{L} \nu+D_{n}(t) \sinh \frac{n \pi}{L} \nu\right) \cos \frac{n \pi}{L} \mu \\
\psi(\mu, \nu, t) & =D_{0}(t)-C_{01}(t) \mu-\sum_{n=1}^{\infty}\left(D_{n}(t) \cosh \frac{n \pi}{L} \nu+C_{n}(t) \sinh \frac{n \pi}{L} \nu\right) \sin \frac{n \pi}{L} \mu,
\end{aligned}
$$

where $A_{n}, B_{n}, C_{n}, D_{n}$ and $C_{01}$ are functions of time to be determined. We can eliminate some of these functions by noting that for a rectangular vessel with a horizontal base at $y=0$, then $y(\mu,-Q, t)=0$ leads to

$$
h_{0}+B_{0}(t)-Q(t)=0, \quad \text { and } \quad B_{n}(t) \cosh \frac{n \pi}{L} Q(t)-A_{n}(t) \sinh \frac{n \pi}{L} Q(t)=0, \quad \text { for } n \geq 1 .
$$

Thus,

$$
\begin{aligned}
& y(\mu, \nu, t)=h_{0}+B_{0}(t)+\nu+\sum_{n=1}^{\infty} A_{n}(t) \frac{\sinh \frac{n \pi}{L}(\nu+Q)}{\cosh \frac{n \pi Q}{L}} \cos \frac{n \pi}{L} \mu, \\
& x(\mu, \nu, t)=\mu+\sum_{n=1}^{\infty} A_{n}(t) \frac{\cosh \frac{n \pi}{L}(\nu+Q)}{\cosh \frac{n \pi Q}{L}} \sin \frac{n \pi}{L} \mu
\end{aligned}
$$

and the conformal modulus is determined via $Q(t)=h_{0}+B_{0}(t)$.

The functions $C_{n}$ and $D_{n}$ in (3.8) and (3.9) can be related to one another by considering the bottom boundary condition (3.3). As the vessel bottom is flat, then on $\nu=-Q(t)$ $y_{\mu}=0$, thus, as we do not want the mapping to be degenerate, i.e. $J=x_{\mu}^{2}+y_{\mu}^{2} \neq 0$, $x_{\mu} \neq 0$ for any $\mu$, we can write the bottom boundary condition for $x \in[0, L]$ as

$$
\psi_{\mu}=-2 \dot{\theta} x_{\mu}\left(x+d_{1}\right)
$$

which on integrating with respect to $\mu$ becomes

$$
\psi=-\dot{\theta}\left(x+2 d_{1}\right) x, \quad \text { on } \quad \nu=-Q(t) .
$$

The time-dependent integration function can be set to zero without loss of generality as the final analysis shows that $\psi$ always appears differentiated with respect to $\mu$ in the governing equations. Similarly if we consider the bottom boundary condition for $\mu \in[L, 2 L]$ then we can write

$$
\psi=\left\{\begin{array}{ll}
-\dot{\theta}\left(x+2 d_{1}\right) x & \text { for } \quad \mu \in[0, L] \\
\dot{\theta}\left(2 L-x+2 d_{1}\right)(2 L-x) & \text { for } \quad \mu \in[L, 2 L]
\end{array}, \quad \text { on } \nu=-Q(t) .\right.
$$


This condition ensures that $\psi$ is an odd function of $\mu$, and so can be expressed as a Fourier Sine series

$$
\psi=\sum_{n=1}^{\infty} \chi_{n} \sin \frac{n \pi}{L} \mu, \quad \text { where } \quad \chi_{n}=-\frac{2 \dot{\theta}}{L} \int_{0}^{L}\left[x(\mu,-Q, t)+2 d_{1}\right] x(\mu,-Q, t) \sin \frac{n \pi}{L} \mu d \mu .
$$

Thus by equating this expression to (3.9) evaluated on $\nu=-Q(t)$ we find that

$$
D_{n}=C_{n} \tanh \frac{n \pi Q}{L}-\frac{\chi_{n}}{\cosh \frac{n \pi Q}{L}}
$$

and $C_{01}=0$. Therefore

$$
\begin{aligned}
\phi(\mu, \nu, t) & =C_{0}(t)+\sum_{n=1}^{\infty}\left(C_{n}(t) \frac{\cosh \frac{n \pi}{L}(\nu+Q)}{\cosh \frac{n \pi Q}{L}}-\chi_{n} \frac{\sinh \frac{n \pi}{L} \nu}{\cosh \frac{n \pi Q}{L}}\right) \cos \frac{n \pi}{L} \mu \\
\psi(\mu, \nu, t) & =D_{0}(t)+\sum_{n=1}^{\infty}\left(-C_{n}(t) \frac{\sinh \frac{n \pi}{L}(\nu+Q)}{\cosh \frac{n \pi Q}{L}}+\chi_{n} \frac{\cosh \frac{n \pi}{L} \nu}{\cosh \frac{n \pi Q}{L}}\right) \sin \frac{n \pi}{L} \mu
\end{aligned}
$$

and on the free-surface, $\nu=0$, the expressions for the variables $x, y, \phi, \psi$ become

$$
\begin{aligned}
Y(\mu, t) & =h_{0}+B_{0}(t)+\sum_{n=1}^{\infty} A_{n}(t) \tanh \frac{n \pi Q}{L} \cos \frac{n \pi}{L} \mu, \\
X(\mu, t) & =\mu+\sum_{n=1}^{\infty} A_{n}(t) \sin \frac{n \pi}{L} \mu \\
\Phi(\mu, t) & =C_{0}(t)+\sum_{n=1}^{\infty} C_{n}(t) \cos \frac{n \pi}{L} \mu, \\
\Psi(\mu, t) & =D_{0}(t)+\sum_{n=1}^{\infty}\left(-C_{n}(t) \tanh \frac{n \pi Q}{L}+\frac{\chi_{n}}{\cosh \frac{n \pi Q}{L}}\right) \sin \frac{n \pi}{L} \mu .
\end{aligned}
$$

In the nonlinear boundary conditions (3.2)-(3.5) $\psi$ and $\Psi$ appear only as derivatives with respect to $\mu$, and so without loss of generality we can set $D_{0}(t)=0$. These expressions give the mappings required to map between the conjugate functions $X$ and $Y$ and $\Phi$ and $\Psi$ on the free-surface for a given value of $t$.

Using the theory and notation of $\S 3$ of Turner and Bridges (2015) these mappings can be written in operator form (full details are given in that paper and here we just record the relevant results). The mapping between $X$ and $Y$ is given by

$$
X=\mu+\mathbf{T}_{q}^{-1}(Y)
$$

where the operator $\mathbf{T}_{q}^{-1}(P)$ acting on $P$, which has Fourier series representation

$$
P(\mu)=\frac{a_{0}}{2}+\sum_{n=1}^{\infty}\left(a_{n} \cos \left(\frac{n \pi}{L} \mu\right)+b_{n} \sin \left(\frac{n \pi}{L} \mu\right)\right)
$$

is

$$
\mathbf{T}_{q}^{-1}(P)=\sum_{n=1}^{\infty} \operatorname{coth}\left(\frac{n \pi Q}{L}\right)\left(-b_{n} \cos \left(\frac{n \pi}{L} \mu\right)+a_{n} \sin \left(\frac{n \pi}{L} \mu\right)\right)
$$


This is equivalent to the conjugate function mapping for the free-sloshing problem considered in Turner and Bridges (2015).

The mapping between $\Phi$ and $\Psi$ however, is different due to the added rotational component. We represent this transformation as

$$
\Psi=\mathbf{T}_{q}(\Phi)+\mathbf{T}_{\chi}(x) \quad \text { or } \quad \Phi=\mathbf{T}_{q}^{-1}\left(\Psi-\mathbf{T}_{\chi}(x)\right),
$$

where

$$
\mathbf{T}_{\chi}(x)=\sum_{n=1}^{\infty} \frac{\chi_{n}}{\cosh \frac{n \pi Q}{L}} \sin \frac{n \pi}{L} \mu,
$$

and $\chi_{n}$ is given in (3.11). Thus the operator $\mathbf{T}_{\chi}(x)$ takes as an input the function $-\dot{\theta}\left(x+2 d_{1}\right) x$ with $x$ evaluated on the bottom of the vessel.

Therefore to determine the time evolution of the free-surface we solve the two freesurface boundary conditions (3.4) and (3.5), using (3.16) and (3.17) to remove the dependence on two of the variables. This is equivalent to the method of Turner and Bridges (2015) and in fact, (3.16) and (3.17) are equivalent to their mappings when $\chi_{n} \equiv 0$ for all $n$.

\subsection{Explicit form of the governing free-surface equations}

The implicit free-surface equations (3.4) and (3.5) can be expressed in explicit form by the following construction. The free-surface $(X(\mu, t), Y(\mu, t))$ is a regular parameterized curve in the plane for each fixed value of $t$. Hence any vector in $\mathbb{R}^{2}$ can be represented uniquely as a linear combination of its normal and tangential vectors. Hence $\left(X_{t}, Y_{t}\right)$ can be expressed as

$$
\left(\begin{array}{c}
X_{t} \\
Y_{t}
\end{array}\right)=\frac{\alpha(\mu, t)}{J}\left(\begin{array}{c}
X_{\mu} \\
Y_{\mu}
\end{array}\right)+\frac{\beta(\mu, t)}{J}\left(\begin{array}{c}
-Y_{\mu} \\
X_{\mu}
\end{array}\right)
$$

where $\alpha(\mu, t)$ and $\beta(\mu, t)$ are functions to be determined. Substituting this into (3.4) gives $\beta=-\Psi_{\mu}-2 \dot{\theta} X_{\mu}\left(X+d_{1}\right)$, but $\alpha$, which represents the tangential fluid velocity at the free-surface, is arbitrary. Hence the kinematic condition can be expressed as

$$
\begin{aligned}
& X_{t}=\left(\frac{\Psi_{\mu}}{J}+\frac{2 \dot{\theta} X_{\mu}}{J}\left(X+d_{1}\right)\right) Y_{\mu}+\frac{\alpha}{J} X_{\mu}, \\
& Y_{t}=-\left(\frac{\Psi_{\mu}}{J}+\frac{2 \dot{\theta} X_{\mu}}{J}\left(X+d_{1}\right)\right) X_{\mu}+\frac{\alpha}{J} Y_{\mu} .
\end{aligned}
$$

From these equations it is clear that $\alpha=X_{\mu} X_{t}+Y_{\mu} Y_{t}$ which can be substituted into (3.5) giving

$$
\begin{aligned}
\Phi_{t}= & -\frac{1}{2 J}\left(\Phi_{\mu}^{2}-\Psi_{\mu}^{2}\right)+2 \dot{\theta} Y_{\mu}\left(X+d_{1}\right) \frac{\Phi_{\mu}}{J}+\ddot{\theta}\left(Y+d_{2}\right)\left(X+d_{1}\right) \\
& +\frac{\dot{\theta}^{2}}{2}\left[-3\left(X+d_{1}\right)^{2}+\left(Y+d_{2}\right)^{2}\right]-g(X \sin \theta+Y \cos \theta)+\frac{\Phi_{\mu}}{J} \alpha(\mu, t) .
\end{aligned}
$$

In (3.18) and (3.19) the function $\alpha(\mu, t)$ is determined such that the mapping (3.16) holds. 


\subsection{Using $\alpha$ to enforce analyticity}

The function $\alpha$ can be determined explicitly in terms of free-surface variables by considering the boundary values of the analytic function $z(\zeta, t)$ where $\zeta=\mu+\mathrm{i} \nu$. We denote the boundary values of this function as $Z(\mu, t)=X(\mu, t)+\mathrm{i} Y(\mu, t)$ and we note that on the boundary

$$
\left.\operatorname{Im}\left(\frac{z_{t}}{z_{\mu}}\right)\right|^{\nu=0}=-\frac{1}{J}\left(\Psi_{\mu}+2 \dot{\theta} X_{\mu}\left(X+d_{1}\right)\right) \quad \text { and }\left.\operatorname{Im}\left(\frac{z_{t}}{z_{\mu}}\right)\right|_{\nu=-Q(t)}=0
$$

and hence the real part of $z_{t} / z_{\mu}$ is determined by the Hilbert-Garrick transform highlighted in Turner and Bridges (2015). In complex form we have

$$
Z_{t}=\frac{1}{J}(\alpha+\mathrm{i} \beta) Z_{\mu}, \quad \text { where } \beta=-\left(\Psi_{\mu}+2 \dot{\theta} X_{\mu}\left(X+d_{1}\right)\right),
$$

and therefore the Hilbert-Garrick transformation gives

$$
\frac{\alpha}{J}=\bar{\alpha}+\mathbf{T}_{q}^{-1}\left(\frac{\beta}{J}\right)=\bar{\alpha}-\mathbf{T}_{q}^{-1}\left(\frac{\Psi_{\mu}}{J}+\frac{2 \dot{\theta} X_{\mu}}{J}\left(X+d_{1}\right)\right),
$$

where $\bar{\alpha}$ is the mean value of $\alpha / J$.

The function $\bar{\alpha}$ is not in general arbitrary, it is related to the time-dependent mean part of $X(\mu, t)$. This problem has been addressed for the classic water wave problem by Choi and Camassa (1999). The time-dependent part of $X(\mu, t)$ would consist of a function $x_{0}(t)$ added to (3.7) and Choi and Camassa (1999) note that $x_{0}$ and $\bar{\alpha}$, in our notation, are related to one another by taking the mean of the first equation of (3.18), and that either of these functions can be considered arbitrary, hence implying the other. This is the strategy we use here. Taking the mean of the first equation of (3.18) leads to

$$
\dot{x}_{0}(t)=F(t) x_{0}+\bar{\alpha}(t),
$$

for a flat bottomed vessel, where $F(t)$ is some time dependent function, whose form is not important to this study. See Appendix A of Turner and Bridges (2015) for how to construct this result. Thus, as we have set $x_{0}=0$ in (3.7) this implies $\bar{\alpha}=0$. Therefore, the forms of (3.18)-(3.19) we solve are

$$
\begin{aligned}
X_{t} & =\frac{\Psi_{\mu}}{J} Y_{\mu}-\mathbf{T}_{q}^{-1}\left(\frac{\Psi_{\mu}}{J}+\frac{2 \dot{\theta} X_{\mu}}{J}\left(X+d_{1}\right)\right) X_{\mu}, \\
Y_{t} & =-\frac{\Psi_{\mu}}{J} X_{\mu}-\mathbf{T}_{q}^{-1}\left(\frac{\Psi_{\mu}}{J}+\frac{2 \dot{\theta} X_{\mu}}{J}\left(X+d_{1}\right)\right) Y_{\mu}, \\
\Phi_{t} & =-g(Y-\delta)-\frac{1}{2 J} \Phi_{\mu}^{2}+\frac{1}{2 J} \Psi_{\mu}^{2}-\mathbf{T}_{q}^{-1}\left(\frac{\Psi_{\mu}}{J}+\frac{2 \dot{\theta} X_{\mu}}{J}\left(X+d_{1}\right)\right) \Phi_{\mu} .
\end{aligned}
$$

\subsection{Numerical scheme}

The numerical scheme used to solve for the time evolution of the free surface is very similar to the pseudo-spectral approach laid out in Turner and Bridges (2015). In this paper we 
briefly highlight the main aspects of the scheme, and the reader is directed to Turner and Bridges (2015) for full details. The free surface equations (3.22) and (3.23) and the vessel equation (2.15) are integrated forward in time using a fourth order Runge-Kutta scheme with a time step $\Delta t$ and specified initial conditions

$$
Y(\mu, 0)=Y_{0}(\mu), \quad X(\mu, 0)=X_{0}(\mu), \quad \Phi(\mu, 0)=\Phi_{0}(\mu), \quad \Psi(\mu, 0)=\Psi_{0}(\mu), \quad \theta(0)=\theta_{0} .
$$

Collocation is used to discretize the interval $\mu \in[0,2 L]$ using $2 N$ collocation points defined by

$$
\mu_{k}=(k-1) \frac{L}{N}, \quad k=1, \ldots, 2 N,
$$

and derivatives are approximated using central differences. By discretising this way we retain $N$ terms in the summations in (3.12)-(3.15).

The initial forms of $X_{0}(\mu)$ and $Y_{0}(\mu)$ are found by solving the nonlinear expression

$$
y=h(x, 0)=H(x) \quad \text { on } \quad \nu=0,
$$

where $H(x)$ is some given initial free-surface profile, found by evaluating at $\mu_{k}$ for $k=$ $1, \ldots, N+1$ and solving the resulting system of $N+1$ simultaneous nonlinear equations via Newton's method. The form of $\Phi_{0}(\mu)$ is stipulated and $\Psi_{0}(\mu)$ is found via (3.15).

We take advantage of the fact that $X(\mu, t)$ and $Y(\mu, t)$ are conjugate functions and integrate only the $Y_{t}$ and $\Phi_{t}$ equations updating $X$ and $\Psi$ via (3.16) and (3.17). The time integration of $(3.22),(3.23)$ and $(2.15)$ proceeds as follows. Firstly we update $\theta$ via (2.15), then we determine $\alpha(\mu, t)=-J \mathbf{T}_{q}\left(\Psi_{\mu} / J+2 \dot{\theta} X_{\mu}\left(x+d_{1}\right) / J\right)$. Now (3.22) and (3.23) are time stepped and (3.16) and (3.17) are used to determine $X$ and $\Psi$ respectively at the new time step. Finally we update the conformal modulus $Q(t)$. This needs to be done iteratively because the process for finding $X(\mu, t)$ and $\Psi(\mu, t)$ depend on $Q(t)$. The conformal modulus is updated via

$$
Q^{(n)}(t)=Y_{0}^{(n)}(t)
$$

where $Y_{0}(t)$ is the non-periodic coefficient of the Fourier transform of $Y(\mu, t)$ and $(n)$ is the number of iterations. This process continues until the relative error in $Q^{(n)}(t)$ is less than $10^{-10}$ for the results in $\S 4$.

The results in $\S 4$ are presented with a time step $\Delta t=5 \times 10^{-5}$ and with $N=400$. The nonlinear terms in the governing equations are de-aliased, and we apply a filter to $X, Y, \Phi$ and $\Psi$ after each complete time step to suppress growing higher order Fourier modes not removed by the de-aliasing. These additional higher order modes occur due to the highly nonlinear form of (3.22) and (3.23). The reason we are not able to fully de-alias the nonlinear terms, is because the equations contain multiplications by the inverse of a finite Fourier expansion, which itself has an infinite Fourier expansion, and so de-aliasing cannot occur completely. However, testing the numerical scheme for various values of $N$ and different de-aliasing values we found that de-aliasing each quadratic nonlinearity using the $2 N / 3$ approach, and applying the additional filtering, to be sufficient to produce converged results.

All results in $\S 4$ are found to be graphically independent of larger $N$ and smaller $\Delta t$. The convergence of the simulations as $N$ is increased can be seen in table 1 which gives values of

$$
I(\Delta t, N)=\int_{0}^{50} \theta d t
$$


for $\Delta t=5 \times 10^{-4}$ and $\Delta t=5 \times 10^{-5}$ for various values of $N$ for the nonlinear simulation result given in figure 8 of $\S 4.3$. The results in table 1 show that the results converge

\begin{tabular}{c|c|c}
$N$ & $\Delta t=5 \times 10^{-4}$ & $\Delta t=5 \times 10^{-5}$ \\
\hline 100 & $9.4782 \times 10^{-3}$ & $10.4267 \times 10^{-3}$ \\
200 & $-6.5894 \times 10^{-3}$ & $-5.1442 \times 10^{-3}$ \\
300 & $-6.6268 \times 10^{-3}$ & $-6.6231 \times 10^{-3}$ \\
400 & $-6.6334 \times 10^{-3}$ & $-6.6088 \times 10^{-3}$ \\
500 & $-6.6375 \times 10^{-3}$ & $-6.6127 \times 10^{-3}$ \\
\hline
\end{tabular}

Table 1: Table of values for $I(\Delta t, N)$ for $\Delta t=5 \times 10^{-4}$ and $\Delta t=5 \times 10^{-5}$ for $N=$ 100, 200, 300, 400 and 500 for the nonlinear simulation result given in figure 8 of $§ 4.3$.

quickly for each $\Delta t$ and have converged by $N=400$. The small differences in the value for $I(\Delta t, N)$ for large $N$ is due to the filtering of the results as discussed above. The forms of $\theta(t)$ for the $N=400$ and $N=500$ results for each $\Delta t$ in table 1 are indistinguishable to graphical accuracy, as are the free-surface elevation results at both walls, $Y(0, t)$ and $Y(L, t)$. Even though results with $N=400$ for both the time steps given in table 1 are equivalent to graphical accuracy, we choose to use the smaller time step as we find this helps with the convergence of the iterative part of the numerical scheme for simulations which contain more nonlinear free-surface profiles. Simulations at this resolution take approximately 120 hours of wall time on a standard desktop machine to run.

\section{Numerical results}

In this section we present results of the nonlinear simulations, highlighting the time evolution of the fluid and vessel motions, the role of initial conditions, as well as the time evolution of the energy budget. For the initial conditions we use the linear expressions for $\phi, h$ and $\theta$ given in (2.16), (2.17) and (2.18), with $\widehat{h}$ and $\widehat{\phi}$ given in (3.12) and (3.13) of Turner et al. (2015). Therefore, $\theta(0)=\theta_{0}=\widehat{\theta}$ and $Y_{0}(\mu)$ and $X_{0}(\mu)$ are found by solving (3.24) with

$$
H(x)=h(x, 0)=h_{0}+\sum_{n=1}^{N} \frac{\widehat{\theta} p_{n}}{\Omega_{r}-g \alpha_{n} T_{n}}\left[\frac{2 \Omega_{r}^{2}}{C_{n}}-T_{n} \alpha_{n}\left(g-\Omega_{r}^{2}\left(h_{0}+d_{2}\right)\right)\right] \cos \left(\alpha_{n} x\right),
$$

where $\omega=\Omega_{r}+\mathrm{i} \Omega_{i}$ is used so we can consider both stable and unstable solutions, $\alpha_{n}=(2 n+1) \pi / L$ and $p_{n}=-4 /\left(L \alpha_{n}^{2}\right)$. Also using (3.14) and (3.15), we have

$$
\begin{aligned}
\Phi_{0}(\mu) & =-\sum_{n=1}^{N} \frac{\Omega_{i} \widehat{\theta} p_{n}}{\Omega_{i}^{2}+g \alpha_{n} T_{n}}\left[g-\frac{2 g}{C_{n}}+\Omega_{i}^{2}\left(h_{0}+d_{2}\right)\right] \cos \left(\alpha_{n} x\right), \\
\Psi_{0}(\mu) & =\sum_{n=1}^{N}\left[\frac{\Omega_{i} \widehat{\theta} p_{n} T_{n}}{\Omega_{i}^{2}+g \alpha_{n} T_{n}}\left[g-\frac{2 g}{C_{n}}+\Omega_{r}^{2}\left(h_{0}+d_{2}\right)\right]+\frac{2 \Omega_{r} \widehat{\theta} p_{n}}{\alpha_{n} C_{n}}\right] \sin \left(\alpha_{n} x\right),
\end{aligned}
$$

and from $(2.18)$

$$
\theta(t)=\widehat{\theta} e^{-\mathrm{i} \Omega_{i} t} \cos \Omega_{r} t
$$


in the linear regime. For the majority of the results in this section we consider the fundamental anti-symmetric sloshing mode as the initial condition to the numerical simulations, other higher frequency sloshing modes could be considered by using different roots of (2.19). This was done in order to validate the numerical scheme but the results are not presented here. Note that the dimensional and non-dimensional frequencies are related via $\Omega_{r}+\mathrm{i} \Omega_{i}=2 \sqrt{g h_{0}}\left(S_{r}+\mathrm{i} S_{i}\right) / L$, where $s=S_{r}+\mathrm{i} S_{i}$ are the roots of $(2.19)$.

\subsection{Simulations close to the linear stability contour}

Here we consider nonlinear simulations either side of the neutral stability contour

$$
G_{0}=\frac{3(1+R)^{2}}{1+6 \delta^{2}}
$$

which comes from the linear theory of Turner et al. (2015). Here $\delta=h_{0} / L$ is the fluid

(a)

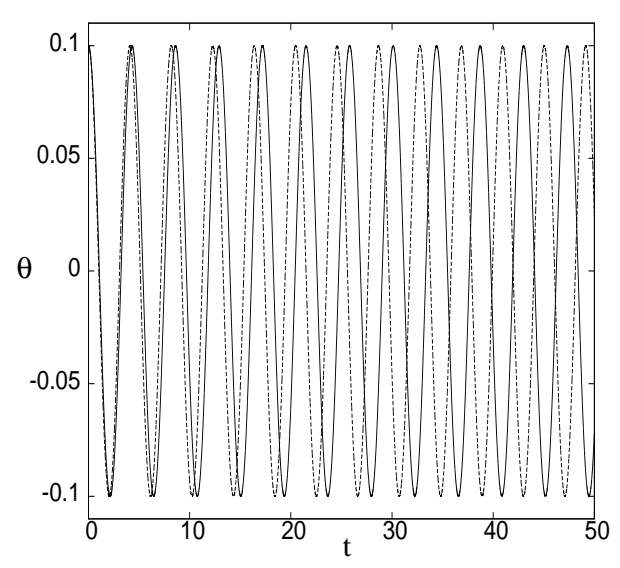

(c)

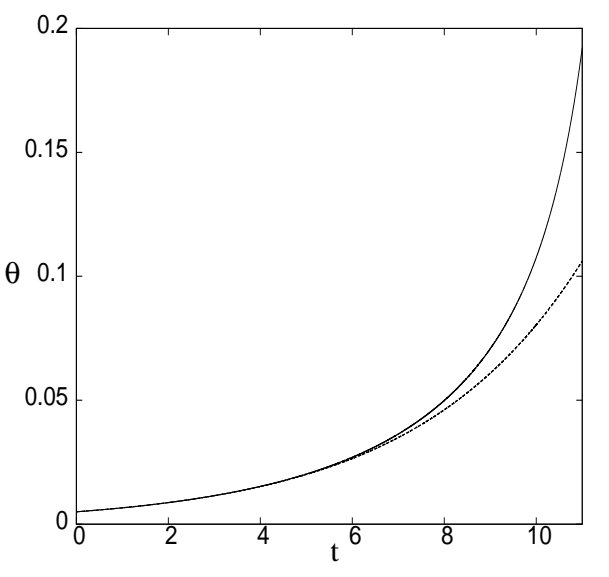

(b)

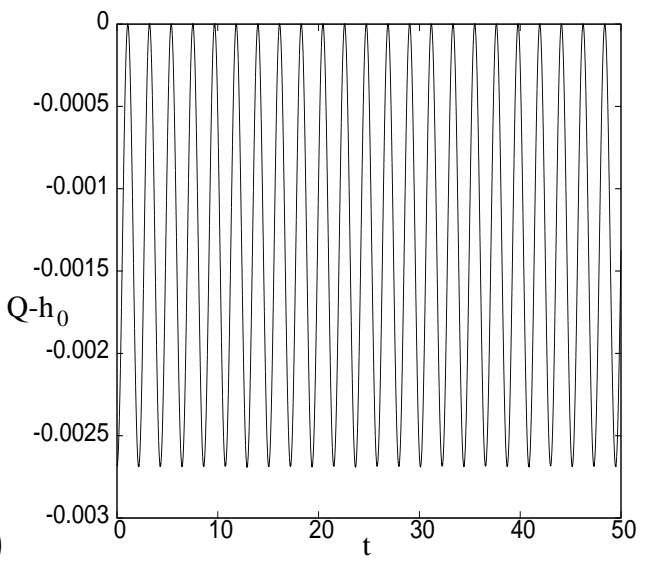

(d)

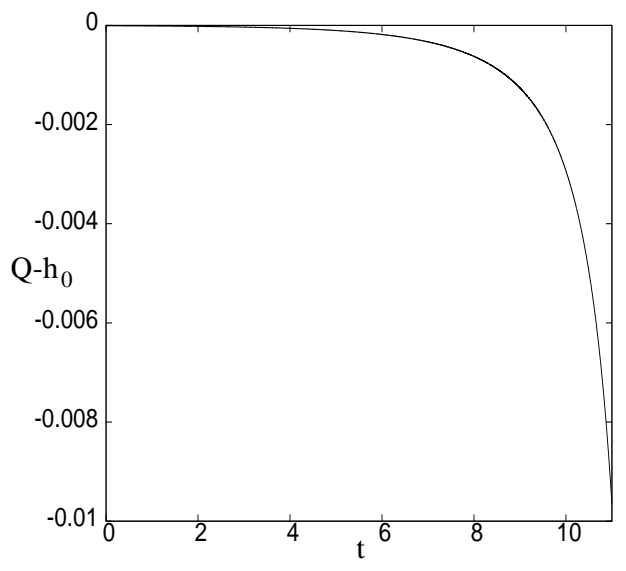

Figure 4: Plot of $\theta(t)$ and $Q(t)-h_{0}$ for the numerical simulations with $\delta=0.2, R=0.5$ and (a,b) $G=5.25, S_{r}=0.3968$ (stable solution) and (c,d) $G=5.45, S_{i}=0.0718$ (unstable solution). In panels (a) and (c) the small amplitude solution (dashed line) is scaled to give the same $\theta$ value at $t=0$ as the larger amplitude solution (solid line).

depth to width ratio and $R=m_{v} / m_{f}$ is the vessel/fluid mass ratio. If $G<G_{0}$ the flow is linearly stable and $\omega=\Omega_{r}$, while if $G>G_{0}$ the flow is linearly unstable and $\omega= \pm \mathrm{i} \Omega_{i}$ with the negative solution corresponding to the unstable mode and the positive solution 
giving the stable mode. Here we consider simulations of the nonlinear system either side of this contour to highlight the effect of nonlinearity on these results. These results are given in figure 4 . For the parameters chosen, $G_{0}=5.44$ so we consider simulations with $G=5.25$ (panels (a) and (b)) and $G=5.45$ (panels (c) and (d)). For each value we show a small amplitude (linear) simulation with $\widehat{\theta}=10^{-4}$ (dashed lines) and a larger amplitude (nonlinear) simulation with $\widehat{\theta}=0.1$ (solid lines). The linearly stable result with $G=5.25$ in figure 4(a) displays periodic behaviour with the linear simulation agreeing exactly with the linear theory (4.1). The nonlinear simulation is also stable and periodic with oscillations between $\theta= \pm 0.1$, but the effect of the nonlinearity acts to decrease the frequency of the oscillations, such that by $t=50$ the linear and nonlinear results are out of phase. Figure 4(b) shows that for the nonlinear solution, $Q(t)-h_{0}$ is oscillating with a frequency which is approximately twice the vessel frequency, which agrees with the asymptotic result from Turner and Bridges (2015) for free sloshing in a rectangular vessel.

When $G=5.45$, we move into the linearly unstable region of parameter space, and the initial condition for the stable simulation which is $\theta \neq 0, h \neq h_{0}$ with $\Phi_{0}=\Psi_{0}=0$ changes to $\theta \neq 0, h \neq h_{0}$ with $\Phi_{0} \neq \Psi_{0} \neq 0$ which induces a moment on the vessel. This can be seen in figure 4(c). Here the angle of the vessel for both the linear and nonlinear simulations keeps increasing until the angle is such that $\theta>\tan ^{-1}(2 \delta)$, and the free-surface touches the bottom of the vessel. At this point the numerical scheme breaks down as the free-surface height goes to zero, and we have not incorporated wetting and drying of the vessel bottom into the numerical scheme for this paper.

The free-surface profiles for both the small and larger amplitude simulations of figure 4 are shown in figure 5 , and close to the neutral curve in the $(R, G)$-plane we observe that the profiles all have a similar appearance, with no visible higher harmonics in the larger amplitude profiles. In fact the free-surfaces appear to be almost straight in these results. However, away from this neutral curve the fluid motion can take on more interesting sloshing motions and free-surface profiles. This is highlighted in $\S 4.3$.

\subsection{Energy budget for simulations}

For the simulations in figure 4 the total amount of energy in the system $E(t)$ is conserved. The sum of potential and kinetic energy is given by

$$
\begin{aligned}
E(t)= & \int_{0}^{L} \int_{0}^{h}\left[\frac{1}{2}\left(u^{2}+v^{2}\right)-\dot{\theta} u\left(y+d_{2}\right)+\dot{\theta} v\left(x+d_{1}\right)+\frac{1}{2} \dot{\theta}^{2}\left(\left(x+d_{1}\right)^{2}+\left(y+d_{2}\right)^{2}\right)\right. \\
& \left.+g\left(\sin \theta\left(x+d_{1}\right)+\cos \theta\left(y+d_{2}\right)\right)\right] \rho d y d x+\frac{1}{2} m_{v}\left[\left(x_{v}+d_{1}\right)^{2}+\left(y_{v}+d_{2}\right)^{2}\right] \dot{\theta}^{2} \\
& +m_{v} g\left(\left(x_{v}+d_{1}\right) \sin \theta+\left(y_{v}+d_{2}\right) \cos \theta\right)-d_{2} g\left(m_{v}+m_{f}\right)
\end{aligned}
$$

and this is shown to be independent of $t$ in Appendix B. However, it is known that the Runge-Kutta integration scheme used in this paper is not energy conserving (Engle et al., 2005), hence in figure 6 we plot the percentage energy error and the total vessel energy

$E_{v}(t)=\frac{1}{2} m_{v}\left[\left(x_{v}+d_{1}\right)^{2}+\left(y_{v}+d_{2}\right)^{2}\right] \dot{\theta}^{2}+m_{v} g\left(\left(x_{v}+d_{1}\right) \sin \theta+\left(y_{v}+d_{2}\right) \cos \theta\right)-d_{2} g\left(m_{v}+m_{f}\right)$,

for the nonlinear stable result from figure 4(a) for the duration of the simulation. Here we see that the maximum percentage error for $E(t)$ is less than $0.3 \%$, which is well within 


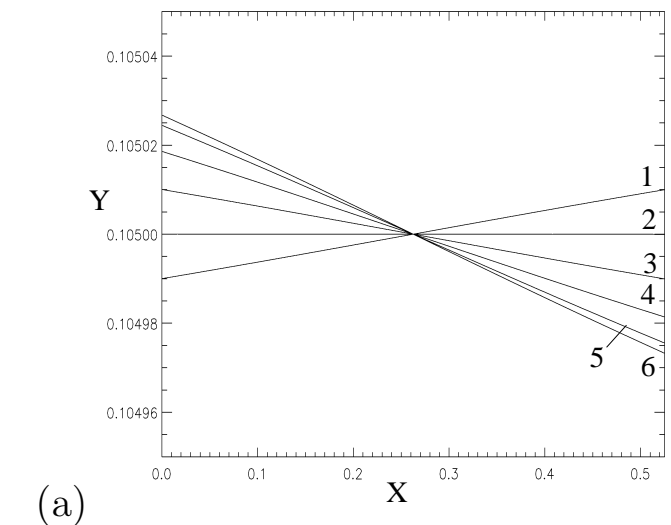

(b)
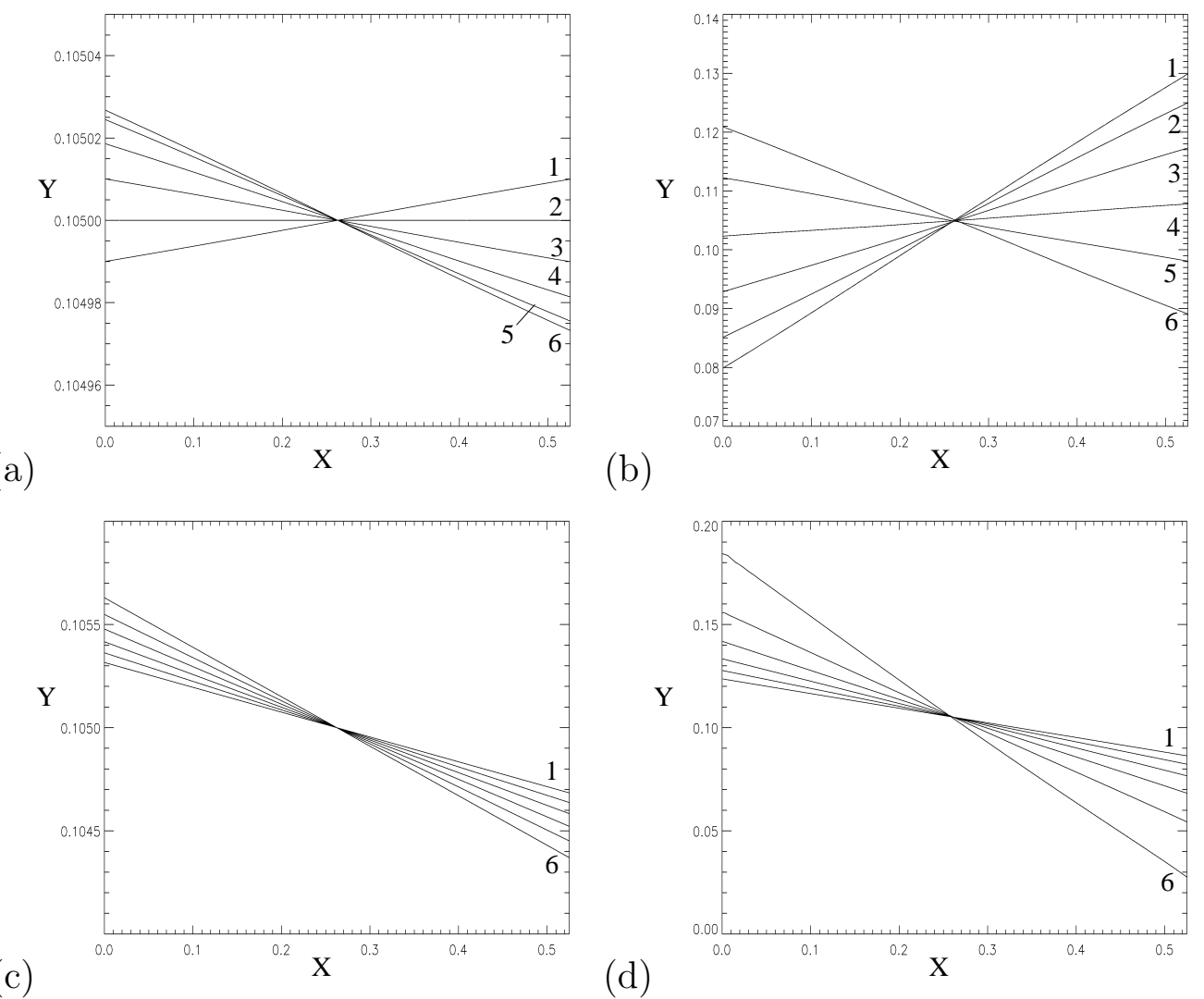

(d)

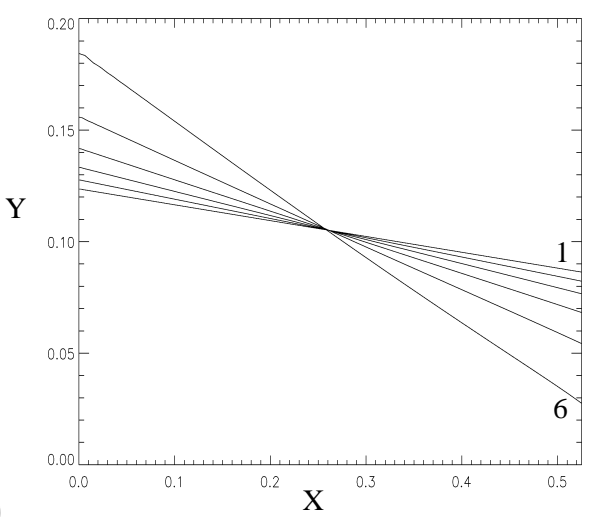

Figure 5: Plot of the free-surface $Y(X, t)$ for the numerical simulations in figure 4. Panels (a) and (b) give the stable solutions for the small amplitude and larger amplitude simulations respectively at $t=11,11.25,11.5,11.75,12$ and 12.25 numbered $1-6$, and panels (c) and (d) give the small amplitude and larger amplitude simulations respectively at $t=9,9.25,9.5,9.75,10$ and 10.25 , here only the first and last profiles are numbered.

(a)

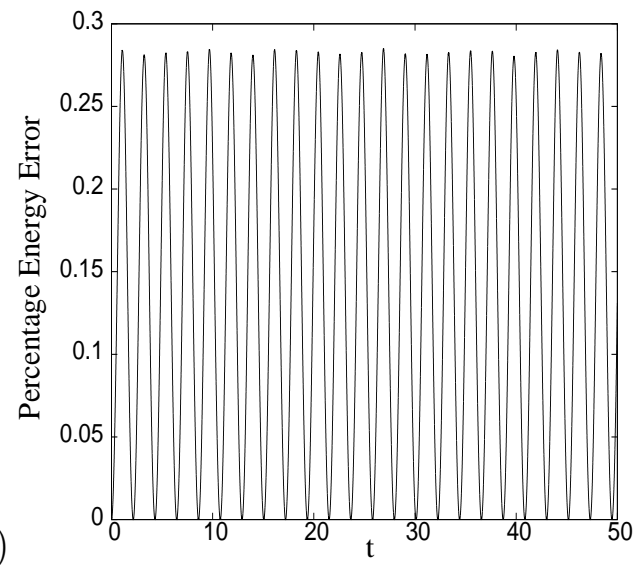

(b)

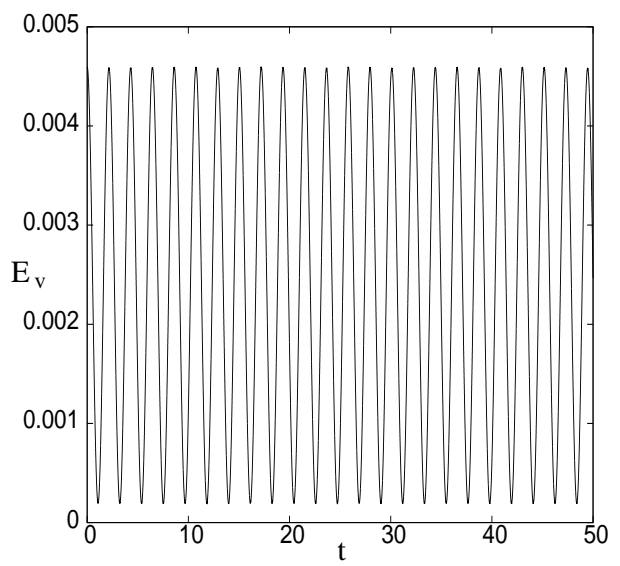

Figure 6: Plot of (a) the percentage energy error $100(E(t)-E(0)) / E(0)$ and (b) $E_{v}$ for the nonlinear result from figure $4(\mathrm{a})$. Note that $E(0)=0.515$.

acceptable computational bounds. Also, the vessel energy is periodic in time, which 
suggests that the energy partition between the vessel and fluid energy is well conserved for these simulations. The percentage energy error is a significant quantity to monitor for the simulations, because knowing that the percentage error is small validates the nonlinear simulations. As stated in $\S 3.4$, the results presented in $\S 4$ are independent of smaller $\Delta t$ and larger $N$, and from (4.2) as $E(t)$ depends on these results, the value of $0.3 \%$ is also independent of smaller $\Delta t$ and larger $N$.

\subsection{Simulations for a variable pole length}

Turner et al. (2015) highlighted for fixed values of $\delta, R$ but varying $G$ (i.e. varying only the pole length) that for small $G$ (long poles) the linear theory predicts that the vessel oscillates with a natural frequency approximately equal to that of a simple pendulum with the fluid motion neglected. But as the pole length decreases the natural frequency of the vessel increases until it reaches a point where the fluid motion in the vessel begins to affect the system frequency. The system frequency then reduces towards zero as the linear neutral stability contour is approached as $G$ is increased further. This behaviour is shown in figure 7 where $S_{r}=L \Omega_{r} /\left(2 \sqrt{g h_{0}}\right)$ is found from the characteristic equation (2.19), and here the circles represent 4 values of $G$ for which we consider the nonlinear evolution for the system in figures 8 and 9 .

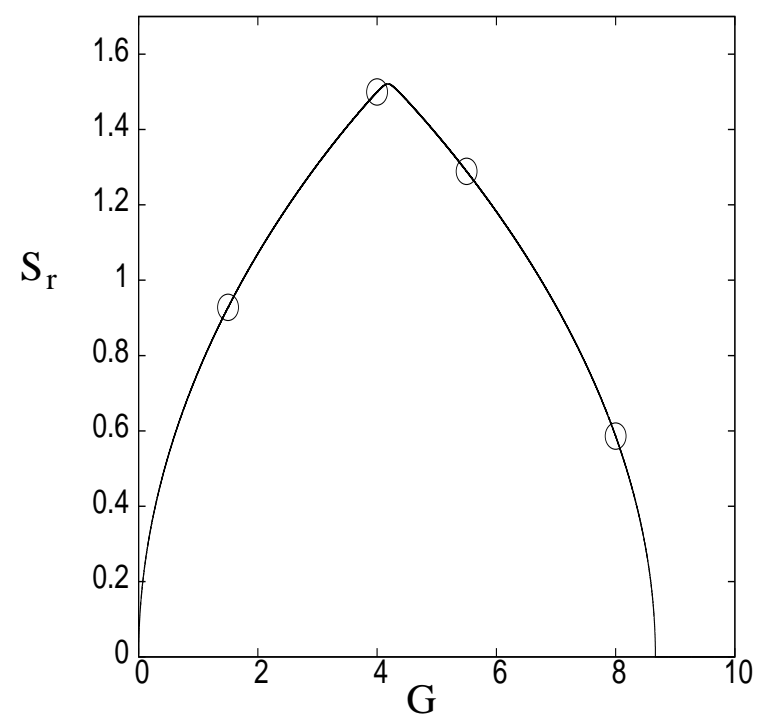

Figure 7: Plot of the 4 values of $G$ considered in figures 8 and 9, and the corresponding values of $S_{r}=L \Omega_{r} /\left(2 \sqrt{g h_{0}}\right)$ for $R=0.75$ and $\delta=0.1$, calculated from the characteristic equation (2.19).

The results for $G=1.5$ show that for the given value of $\widehat{\theta}=0.035$, the $\theta(t)$ evolution is in excellent agreement with the linear result (4.1) (small black dots in left panels of figure 8), and the corresponding value of $Q(t)-h_{0}$ is small at $O\left(10^{-8}\right)$. The reason the result agrees so well with the linear result is clear from figure 9 where we observe that the free-surface variation from $h_{0}$ is small compared to those for larger $G$ values. Hence as the fluid displacement amplitude is small, the fluid motion does not affect the vessel motion, and therefore the vessel motion is approximately linear for this value of $\widehat{\theta}$. 

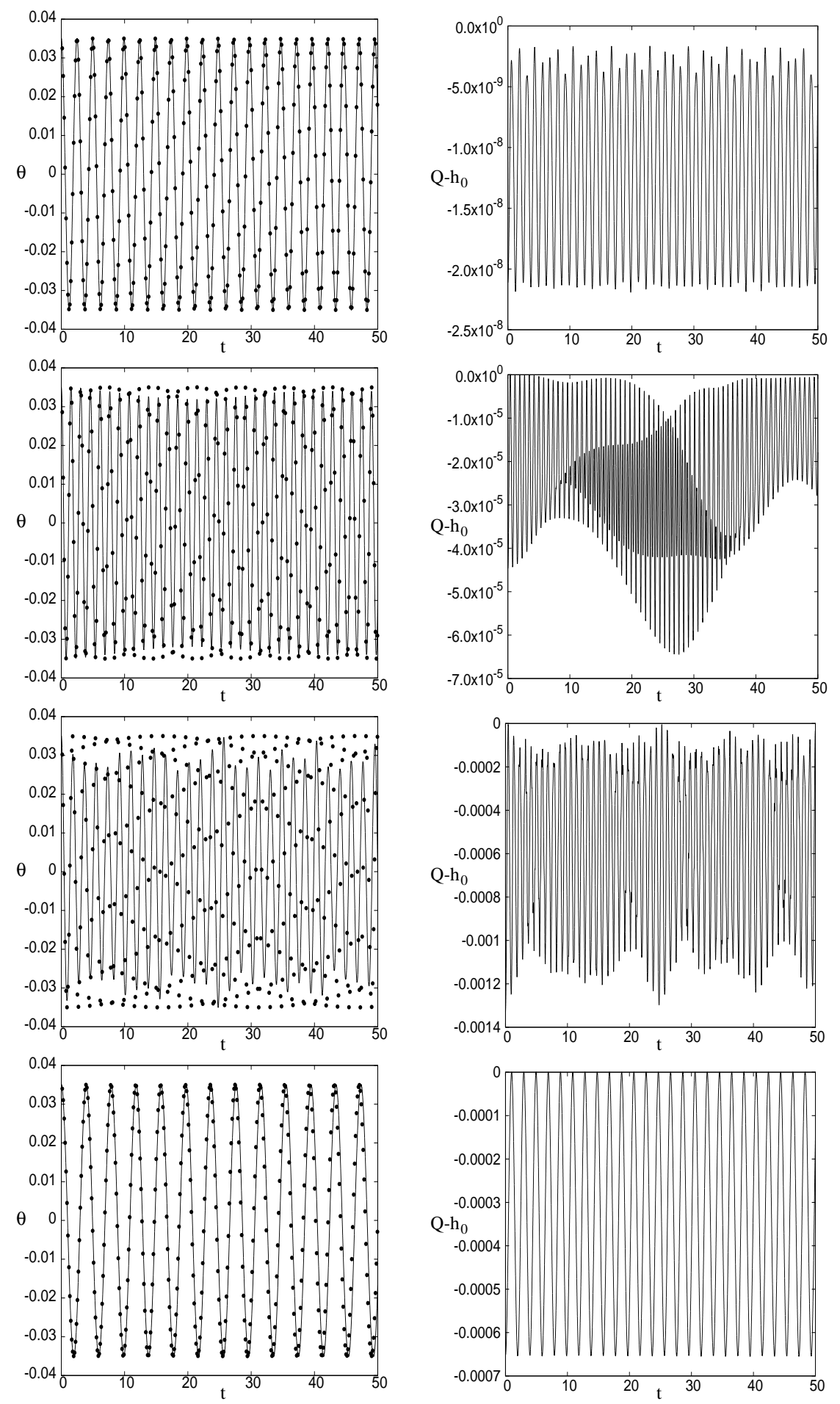

Figure 8: Plot of $\theta(t)$ and $Q(t)-h_{0}$ for the nonlinear simulations with $\delta=0.1, R=0.75$ and $\widehat{\theta}=0.035$. The values of $G$ correspond to those from figure 7 with, from top to bottom, $G=1.5, G=4.0, G=5.5$ and $G=8.0$. The solid circles in the $\theta(t)$ panels represent the linear solution (4.1) with $\Omega_{r}=2 \sqrt{g h_{0}} S_{r} / L$.

When $G$ is increased to 4.0 the $\theta(t)$ motion is again essentially linear, i.e. the frequency agrees with the linear natural frequency, but there is a small variation in the 

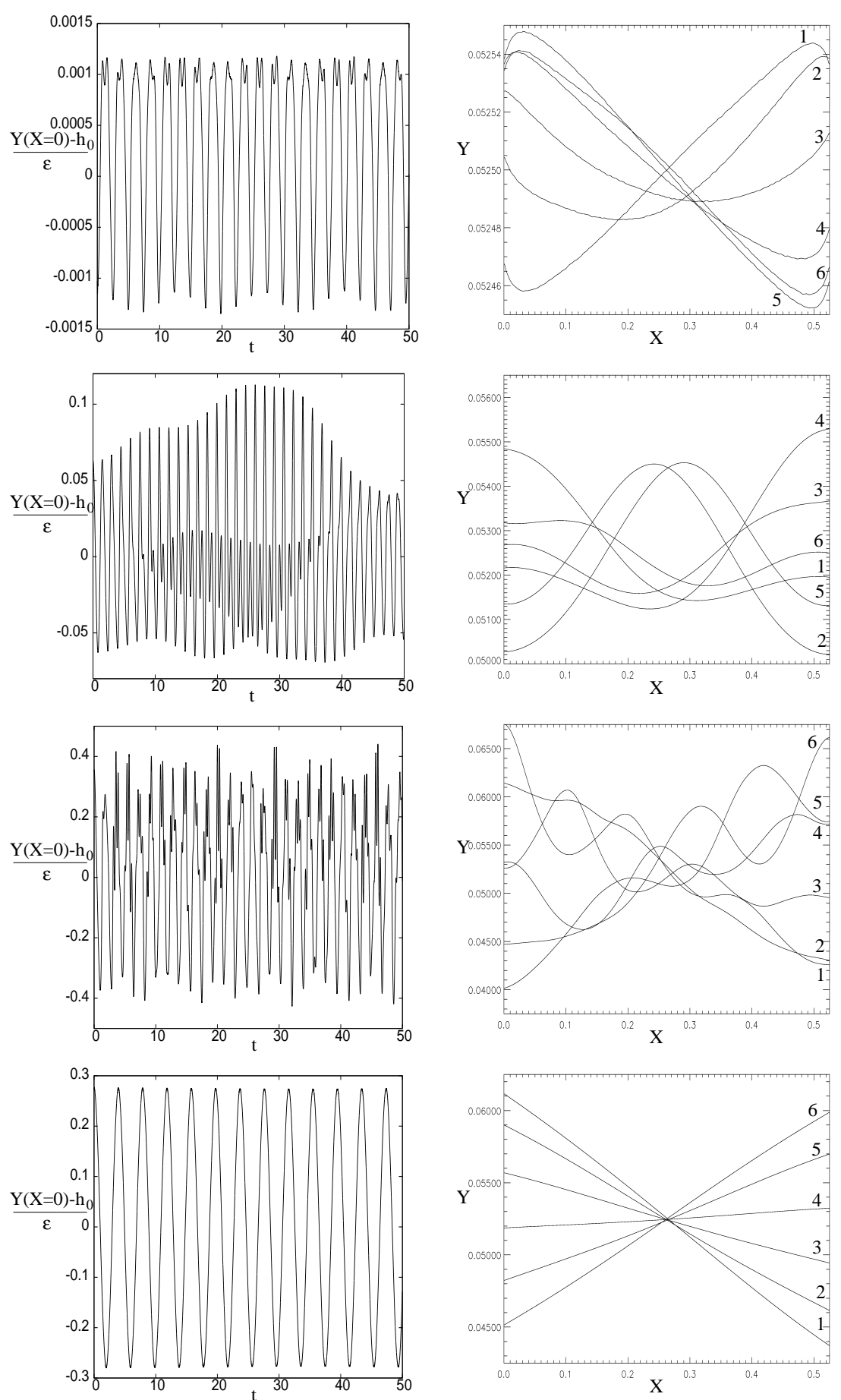

Figure 9: Plot of $Y(X=0, t) / \epsilon$ and $Y(X, t)$ for the nonlinear simulations in figure 8. In the profile panels the results numbered 1-6 correspond to the times $t=$ $20,20.25,20.5,20.75,21$ and 21.25 respectively.

amplitude of the oscillations over a long time-scale. Here the fluid free-surface profiles deviate from $h_{0}$ by a larger magnitude than they did for $G=1.5$ and hence the fluid motion affects the vessel motion more in this case. Increasing $G$ further to 5.5 we find that the free-surface profiles show a highly nonlinear behaviour, and this is reflected in 
large values of $Q(t)-h_{0}$ which contain multiple higher frequencies, which in turn can be observed in $\theta(t)$. Finally at $G=8.0$ we have a similar result to that in figure 4(a) where the amplitude of the $\theta(t)$ oscillations are the same as for the corresponding linear result, but with a reduced frequency. Here again the magnitude of $Q(t)-h_{0}$ is large, but smaller than in the $G=5.5$ case.

Thus for fixed values of $R, \delta$ and $\widehat{\theta}$, reducing the pole length produces results which initially are approximately linear and independent of the fluid motion for very long poles. Those results then pass though highly nonlinear fluid-vessel motions including higher frequency harmonics for moderate pole lengths, and finally back to periodic nonlinear behaviour where the frequency of the vessel is highly coupled to the fluid motion for short poles. The results in figure 9 also show that the magnitude of $Q-h_{0}$ is directly related to the amount of nonlinearity observed in the vessel motion, which agrees with the observation of Turner and Bridges (2015).

The simulation results in figures 8 and 9 are somewhat idealised numerical experiments because the initial conditions are taken from the linear theory. Hence it is reasonable to ask whether similar conclusions can be drawn for initial conditions which could be generated in the experimental setup of figure 2. Hence we repeat the simulations of figures 8 and 9 except we consider the initial conditions

$$
\theta=\widehat{\theta}, \quad \dot{\theta}=0, \quad \Phi_{0}(\mu)=0, \quad \Psi_{0}(\mu)=0,
$$

and $Y_{0}(\mu)$ and $X_{0}(\mu)$ are found by solving (3.24) with

$$
H(x)=h_{0}-\widehat{\theta}\left(x-\frac{L}{2}\right) .
$$

This initial condition corresponds to a vessel which is released from rest with an initial angle $\widehat{\theta}$, and a horizontal free-surface. The resulting $\theta(t)$ and $Q(t)-h_{0}$ evolutions are presented in figure 10.

The main conclusion to draw about each of these simulations, with the exception of the $G=5.5$ case, is they are all more nonlinear than the results in figure 8, with higher frequency harmonics appearing in both $\theta(t)$ and $Q(t)$. The larger amount of nonlinearity is also reflected in the conformal modulus plots, as the variation in $Q-h_{0}$ is now the same order of magnitude for each simulation. Hence, when higher order sloshing modes are present in the initial condition (as is the case for the initial condition (4.3)-(4.4)), the sloshing fluid has a larger effect on the vessel motion, as can been seen for $G=1.5$ and $G=4.0$. In these simulations the variation of $\theta(t)$ for the short pole results are similar to those in figure 8 , but the long pole results $(G=1.5$ and $G=4.0)$ now experience feedback from the fluid motion and thus both results now have a slow time modulation of the vessel oscillation magnitude. Both of these results are significantly different from those results in figure 8 . Hence ensuring the initial conditions for the simulations are correct, is important for accurately modelling the coupled dynamics.

\subsection{Simulations containing similar frequency modes}

Another interesting feature of the system highlighted in the linear study of Turner et al. (2015) is the existence of parameter values where there exists two anti-symmetric sloshing modes with approximately the same natural frequency. To be clear, this is not a double 

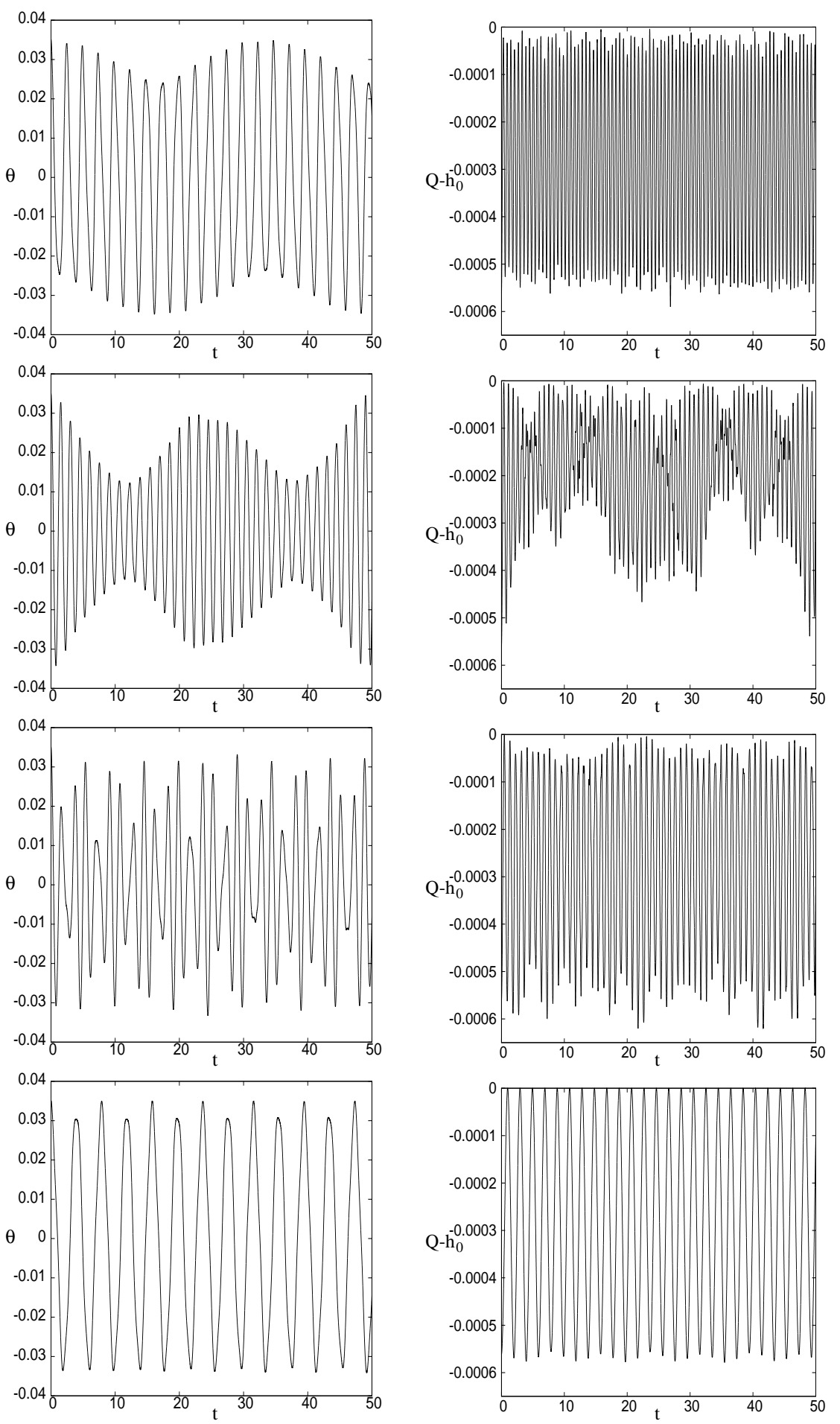

Figure 10: Plot of $\theta(t)$ and $Q(t)-h_{0}$ for the nonlinear simulations from figure 8 , but with the initial condition given by (4.3) and (4.4).

root of (2.19) as one of the roots is removable as they come together. Figure 11 shows the vessel evolution $\theta(t)$, for two parameter sets, which contain two modes with similar natural frequencies. Here the initial condition for the simulations is a superposition of the two linear sloshing modes, both with the same value of $\widehat{\theta}=10^{-5}$ for the small 
(a)

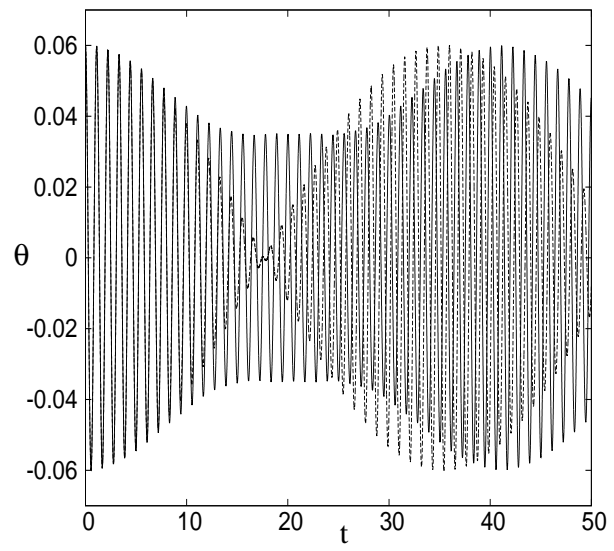

(c)

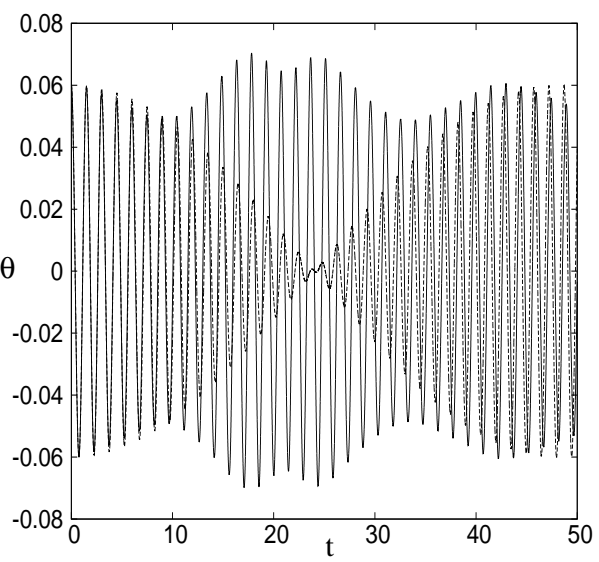

(b)

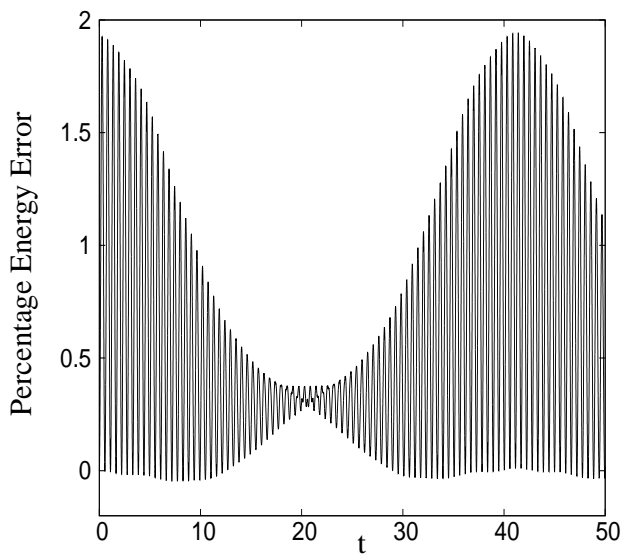

(d)

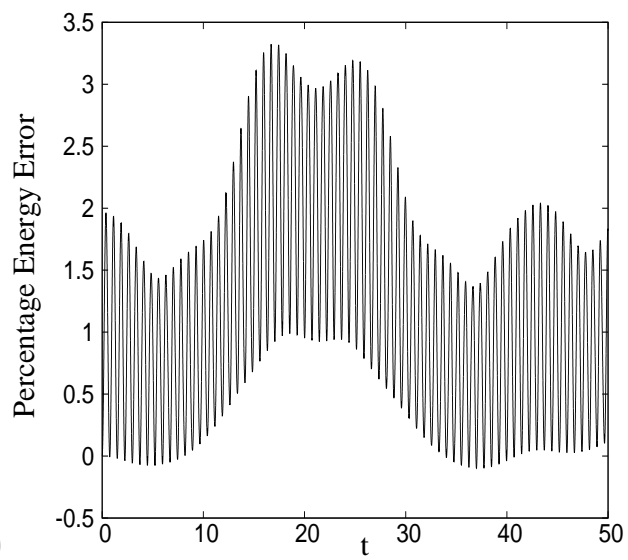

Figure 11: Plot of $\theta(t)$ and the percentage energy error $100(E(t)-E(0)) / E(0)$ for (a), (b) $(\delta, R, G)=(0.2,2,6.8)$ and $(\mathrm{c}),(\mathrm{d})(0.1,0.75,4.3)$. In panels (a) and (c) the solid lines give the nonlinear result with $\widehat{\theta}=0.03$ for each eigenmode, while the dashed line gives the linear result with $\widehat{\theta}=10^{-5}$ for each eigenmode scaled up. In (b) $E(0)=0.179$ and in $(\mathrm{d}) E(0)=0.132$ for the energy error evolutions for the larger amplitude simulations.

amplitude simulations and $\widehat{\theta}=0.03$ for the larger amplitude simulations. The dashed line in both panels (a) and (c) gives the small amplitude (linear) simulation (scaled up for comparison with the larger amplitude (nonlinear) result) and these both show that the small amplitude result exudes a wave pulse behaviour. However, in the large amplitude simulations the region where $\theta$ decays to almost zero between the pulses is reduced, as seen in panel (a), and in fact for some parameter sets, such as that in panel (c), the decay turns into a feedback mechanism around $t=10$ and produces oscillations which are larger than the initial displacement of the vessel. Hence, unlike the single mode initial condition, nonlinear results in figure 8, where the vessel oscillations are bounded by the initial angular displacement, the two frequency simulations can produce oscillations larger than this initial displacement. This would have implications on the physical system if the vessel was setup to oscillate in a restricted domain with a maximum amplitude only slightly smaller than the dimension of the domain. This would also have significant implications on the slosh-induced-rolling of a ship, as it would mean the ship could readily capsize. It is also worth noting that for these simulations the percentage energy error, panels (b) and (d), is larger than for the single mode cases, but is still only around $3 \%$ 
at its largest, which is within an acceptable numerical tolerance.

\section{Conclusions and Discussion}

This paper presented fully nonlinear numerical results for the pendulum-slosh problem. The numerical scheme to solve the coupled system used a time-dependent conformal mapping to map the physical domain to a rectangle in the computational domain with a time-dependent aspect ratio, known as the conformal modulus, $Q(t)$. The conformal modulus has to be found as part of the solution procedure, making the implementation of the numerical scheme more difficult. It was shown that the magnitude of $\left|Q(t)-h_{0}\right|$, where $h_{0}$ is the equilibrium fluid height, is directly related to the magnitude of the nonlinearity in the system. This result for the dynamic case agrees with the conclusion of Turner and Bridges (2015) for the free-sloshing problem in a fixed rectangular tank.

The results of the nonlinear simulations in the vicinity of the linear neutral stability contour showed that the nature of the solution (i.e. being stable or unstable) is confirmed. Also the stable solution still exhibited a periodic behaviour, except with a reduced frequency compared to the small amplitude (linear) result. Thus in this 'short pole' case the effect of higher order sloshing modes appears to be small when the initial condition is taken to be the fundamental linear sloshing mode.

For moderate length poles however, the results of this study show that nonlinear effects are more significant, and the presence of higher frequency sloshing modes in the fluid can be observed in the vessel motion. For an initial condition which could be easily implemented in an experiment, of a vessel released from rest from a non-zero angle, higher frequency sloshing modes were observed in the vessel motion for all pole lengths considered. Thus, we conclude that the nonlinear evolution of the system is strongly dependent on the form of the initial condition chosen. The initial conditions relevant for a physical experiment consist of a superposition of many sloshing modes which interact more readily with each other than in the case of the single fundamental sloshing mode initial condition.

The nonlinear interaction of the sloshing modes was also demonstrated for system parameter values where the characteristic equation (2.19) exhibits two anti-symmetric sloshing modes with approximately the same natural frequency. With an initial condition comprising of the superposition of these two modes, the nonlinear simulation showed that for some parameter values, the vessel could exhibit oscillations which were larger than the initial $\theta$ displacement of the vessel. This would have drastic implications if the vessel was designed to oscillate within a fixed domain, fractionally larger than the initial displacement of the vessel. This result would also be significant on the slosh-inducedrolling of a ship, because the larger than expected vessel oscillations could lead the ship to capsize.

All the large amplitude simulations presented in this paper had an energy budget which was well conserved by the numerical scheme, to within some allowed tolerance factor, confirming that the numerical approach used here is appropriate for performing simulations of dynamic coupling problems.

The strength of the conformal mapping scheme considered in this paper is that additional aspects of the problem such as different vessel geometries or submerged structures could be easily incorporated. However, due to the iterative nature of the numerical 
scheme, because the conformal modulus has to be found as part of the solution, it is moderately numerically expensive, and hence is really only restricted to two-dimensional problems. If the scheme could be formulated with a PDE for the evolution of $Q(t)$, then iterations would no longer be required and some speed up would be possible.

\section{Acknowledgments}

This work is supported by the EPSRC under grant number EP/K008188/1. Due to confidentiality agreements with research collaborators, supporting data can only be made available to bona fide researchers subject to a non-disclosure agreement. Details of the data and how to request access are available from the University of Surrey publications repository: researchdata@surrey.ac.uk

\section{- Appendix -}

\section{A Parametric boundary conditions}

Here we derive the parametric forms of the fluid boundary conditions in the computational domain. For our mapping we can write

$$
\begin{aligned}
\phi_{x} & =\frac{1}{J} y_{\nu} \phi_{\mu}-\frac{1}{J} y_{\mu} \phi_{\nu}=\frac{1}{J} x_{\mu} \phi_{\mu}+\frac{1}{J} y_{\mu} \psi_{\mu}, \\
\phi_{y} & =-\frac{1}{J} x_{\nu} \phi_{\mu}+\frac{1}{J} x_{\mu} \phi_{\nu}=\frac{1}{J} y_{\mu} \phi_{\mu}-\frac{1}{J} x_{\mu} \psi_{\mu},
\end{aligned}
$$

using the chain rule and the Cauchy-Riemann equations. Thus $\phi_{x}=0$ at $x=0, L$ simply reduces to (3.2) and the bottom boundary condition on $y=0$ becomes

$$
\phi_{y}=\frac{1}{J}\left(y_{\mu} \phi_{\mu}-x_{\mu} \psi_{\mu}\right)=2 \dot{\theta}\left(x+d_{1}\right)
$$

which for a flat bottomed vessel $\left(y_{\mu}=0\right)$ reduces to (3.3).

The kinematic free-surface condition says that

$$
\frac{\partial}{\partial t}(X, Y) \cdot \mathbf{n}=\left(\Phi_{x}, \Phi_{y}-2 \dot{\theta}\left(X+d_{1}\right)\right) \cdot \mathbf{n},
$$

where $\mathbf{n}=J^{-1 / 2}\left(-Y_{\mu} X_{\mu}\right)^{T}$ is the normal to the free-surface. Expanding the dot product then leads to

$$
X_{\mu} Y_{t}-Y_{\mu} X_{t}=-Y_{\mu} \Phi_{x}+X_{\mu}\left(\tilde{\Phi}_{y}-2 \dot{\theta}\left(X+d_{1}\right)\right)
$$

If at this stage we introduce the single valued form of the free-surface $Y=h(\mu, t), X=\mu$, then this equation reduces back to the form of the kinematic free-surface equation in (2.14). Also note that using (A-1) and (A-2) this equation reduces to (3.4). 
Finally, to transform the dynamic boundary condition into parametric form, we use (2.3) from Bridges and Donaldson (2011) and the Cauchy-Riemann equations to write

$$
\phi_{t}(x, y, t)=\phi_{t}(\mu, \nu, t)-\frac{1}{J}\left(y_{\mu} y_{t}+x_{\mu} x_{t}\right) \phi_{\mu}-\frac{1}{J}\left(y_{\mu} x_{t}-x_{\mu} y_{t}\right) \psi_{\mu} .
$$

Substituting this, and the forms of $\phi_{x}$ and $\phi_{y}$ from (A-1) and (A-2) into the dynamic boundary condition and evaluating on the free-surface leads to (3.5).

\section{B Proof of energy conservation for stable solutions}

The simplest way to prove that the system energy (4.2) is conserved is to show that $\frac{d E}{d t}=0$. Therefore by splitting the energy up into individual integrals and bringing the $\theta$ dependence outside of each integral we can write

$$
\begin{aligned}
\frac{d E}{d t}= & \dot{\theta}\left\{\left(m_{v}\left[\left(x_{v}+d_{1}\right)^{2}+\left(y_{v}+d_{2}\right)^{2}\right]+\int_{0}^{L} \int_{0}^{h}\left[\left(x+d_{1}\right)^{2}+\left(y+d_{2}\right)^{2}\right] \rho d y d x\right) \ddot{\theta}\right. \\
& +\dot{\theta} \frac{d}{d t} \int_{0}^{L} \int_{0}^{h}\left[\left(x+d_{1}\right)^{2}+\left(y+d_{2}\right)^{2}\right] \rho d y d x+m_{v} g\left[\left(x_{v}+d_{1}\right) \cos \theta-\left(y_{v}+d_{2}\right) \sin \theta\right] \\
& \left.+g \int_{0}^{L} \int_{0}^{h}\left[\cos \theta\left(x+d_{1}\right)-\sin \theta\left(y+d_{2}\right)\right] \rho d y d x-\frac{d}{d t}\left[\int_{0}^{L} \int_{0}^{h}\left[u\left(y+d_{2}\right)-v\left(x+d_{1}\right)\right] \rho d y d x\right]\right\} \\
& -\frac{\dot{\theta}}{2} \frac{d}{d t} \int_{0}^{L} \int_{0}^{h}\left[\left(x+d_{1}\right)^{2}+\left(y+d_{2}\right)^{2}\right] \rho d y d x+\frac{1}{2} \frac{d}{d t} \int_{0}^{L} \int_{0}^{h}\left(u^{2}+v^{2}\right) \rho d y d x \\
& -\ddot{\theta} \int_{0}^{L} \int_{0}^{h}\left[u\left(y+d_{2}\right)-v\left(x+d_{1}\right)\right] \rho d y d x \\
& +g\left[\sin \theta \frac{d}{d t} \int_{0}^{L} \int_{0}^{h}\left(x+d_{1}\right) \rho d y d x+\cos \theta \frac{d}{d t} \int_{0}^{L} \int_{0}^{h}\left(y+d_{1}\right) \rho d y d x\right] .
\end{aligned}
$$

The quantity in the curly brackets is equal to 0 as it corresponds to the vessel equation (2.8). The other terms are simplified by moving the time derivative inside the integrals using Reynolds transport formula and can be combined to give

$$
\begin{aligned}
\frac{d E}{d t}= & \int_{0}^{L} \int_{0}^{h} u\left[\frac{D u}{D t}-\ddot{\theta}\left(y+d_{2}\right)+g \sin \theta-\left(x+d_{1}\right) \dot{\theta}^{2}\right] \rho d y d x \\
& +\int_{0}^{L} \int_{0}^{h} v\left[\frac{D v}{D t}+\ddot{\theta}\left(x+d_{1}\right)+g \cos \theta-\left(y+d_{2}\right) \dot{\theta}^{2}\right] \rho d y d x .
\end{aligned}
$$

Then via the Euler equations (2.1) and (2.2)

$$
\begin{aligned}
\frac{d E}{d t} & =-\int_{0}^{L} \int_{0}^{h}\left(u \frac{\partial p}{\partial x}+v \frac{\partial p}{\partial y}\right) d y d x \\
& =-\int_{0}^{L} \int_{0}^{h} \mathbf{u} \cdot \nabla p d y d x \\
& =\int_{0}^{L} \int_{0}^{h} p \nabla \cdot \mathbf{u} d y d x+\int_{\text {boundary }} p(\mathbf{u} \cdot \mathbf{n}) d S, \quad \text { where } \mathbf{n} \text { is a unit normal to the fluid, } \\
& =0
\end{aligned}
$$

as the flow is incompressible, and via the boundary conditions (2.3)-(2.5). 


\section{References}

Abramson, H. N., Chu, W. H., and Ransleben, JR., G. E. (1961). Representation of fuel sloshing in cylindrical tanks by an equivalent mechanical model. Am. Rocket Soc., 31, $1697-1705$.

Adee, B. H. and Caglayan, I. (1982). The effects of free water on deck on the motions and stability of vessels. In Proc. Second Inter. Conf. Stab. Ships and Ocean Vehicles, Tokyo. SNAME, Springer.

Alemi Ardakani, H. (2010). Rigid-body motion with interior shallow-water sloshing. PhD Thesis, University of Surrey, Guildford, UK.

Alemi Ardakani, H. and Bridges, T. J. (2010). Dynamic coupling between shallow-water sloshing and horizontal vehicle motion. Europ. J. Appl. Math, 21, 479-517.

Alemi Ardakani, H., Bridges, T. J., and Turner, M. R. (2012). Resonance in a model for Cooker's sloshing experiment. Euro. J. Mech. B/Fluids, 36, 25-38.

Bridges, T. J. and Donaldson, N. M. (2011). Variational principles for water waves from the viewpoint of a time dependent moving mesh. Mathematika, 57, 147-173.

Byatt-Smith, J. (1971). An integral equation for unsteady surface waves and a comment on the boussinesq equation. J. Fluid Mech., 49, 625-633.

Caglayan, I. and Storch, R. L. (1982). Stability of fishing vessels with water on deck: a review. J. Ship Research, 26, 106-116.

Challis, N. and Burley, D. (1982). A numerical method for conformal mapping. IMA Journal of Numerical Analysis, 2(2), 169-181.

Choi, W. and Camassa, R. (1999). Exact evolution equations for surface waves. J. Eng, Mech., 125, 756-760.

Constantin, A., Strauss, W., and Varvaruca, E. (2014). Global bifurcation of steady gravity water waves with critical layers. arXiv, v1, 1407.0092.

Cooker, M. J. (1994). Water waves in a suspended container. Wave Motion, 20, 385-395.

Dillingham, J. (1981). Motion studies of a vessel with water on deck. Wave Motion, 18, $38-50$.

Dyachenko, A. I., Kuznetsov, E. A., Spector, M. D., and Zakharov, V. E. (1996). Analytical description of the free surface dynamics of an ideal fluid (canonical formalism and conformal mapping). Phys. Lett. A, 221, 73-79.

Dyachenko, A. I., Zakharov, V. E., and Kuznetsov, E. A. (1999). Nonlinear dynamics of the free surface of an ideal fluid. Plasma Phys. Rep., 22, 916-928.

Engle, R. D., Skeel, R. D., and Drees, M. (2005). Monitoring energy drift with shadow hamiltonians. Journal of Computational Physics, 206(2), 432-452. 
Faltinsen, O. M. and Timokha, A. N. (2009). Sloshing. Cambridge University Press (Cambridge).

Ferrant, P. and Le Touze, D. (2001). Simulation of sloshing waves in a 3D tank based on a pseudo-spectral method. In Proc. 16th Int. Workshop on Water Waves and Floating Bodies, Hiroshima, Japan.

Frandsen, J. B. (2004). Sloshing motions in excited tanks. J. Comput. Phys., 196, 53-87.

Frandsen, J. B. (2005). Numerical predictions of tuned liquid tank structural systems. J. Fluids \&6 Structures, 20, 309-329.

Gardarsson, S., Yeh, H., and Reed, D. (2001). Behavior of sloped-bottom tuned liquid dampers. Journal of engineering mechanics, 127(3), 266-271.

Hirt, C. W. and Nichols, B. D. (1981). Volume of fluid (VOF) method for the dynamics of free boundaries. Journal of computational physics, 39(1), 201-225.

Ibrahim, R. A. (2005). Liquid Sloshing Dynamics. Cambridge University Press (Cambridge).

Idir, M., Ding, X., Lou, M., and Chen, G. (2009). Fundamental frequency of water sloshing waves in a sloped-bottom tank as tuned liquid damper. In Structures Congress 2009: Dont Mess with Structural Engineers: Expanding Our Role, pages 1-10. ASCE.

Li, Y., Hyman, J., and Choi, W. (2004). A numerical study of the exact evolution equations for surface waves in water of finite depth. Stud. Appl. Math., 113, 303-324.

Moiseev, N. N. (1953). The problem of solid objects containing liquids with a free surface. Mat. Sbornik., 32(74)(1), 61-96 (in Russian).

Moiseyev, N. N. and Rumyantsev, V. V. (1968). Dynamic Stability of Bodies Containing Fluid. Springer-Verlag (New York).

Papamichael, N. and Stylianopoulos, N. (2010). Numerical conformal mapping: Domain decomposition and the mapping of quadrilaterals. World Scientific (Singapore).

Seidl, A. and Klose, H. (1985). Numerical conformal mapping of a towel-shaped region onto a rectangle. SIAM J. Sci. Comput., 6(4), 833-842.

Shamin, R. V. (2009). Dynamics of an ideal liquid with a free surface in conformal variables. J. Math. Sci., 160(5), 537-678.

Telste, J. G. (1985). Calculation of fluid motion resulting from large-amplitude forced heave motion of a two-dimensional cylinder in a free surface. In International Conference on Numerical Ship Hydrodynamics, 4th, pages 81-93.

Turner, M. R. and Bridges, T. J. (2015). Time-dependent conformal mapping of doublyconnected regions. Adv. Comp. Math (submitted).

Turner, M. R., Alemi Ardakani, H., and Bridges, T. J. (2015). Instability of sloshing motion in a vessel undergoing pivoted oscillations. J. Fluid Struct., 52, 166-180. 
Ushijima, S. (1998). Three-dimensional arbitrary lagrangian-eulerian numerical prediction method for non-linear free surface oscillation. International Journal for Numerical Methods in Fluids, 26(5), 605-623.

Vanden-Broeck, J. and Schwartz, L. (1979). Numerical computation of steep gravity waves in shallow water. Phys. Fluids, 22, 1868-1871.

Viotti, C., Dutykh, D., and Dias, F. (2013). The conformal-mapping method for surface gravity waves in the presence of variable bathymetry and mean current. Procedia IUTAM, pages 1-13.

Whitney, A. K. (1971). The numerical solution of unsteady free surface flows by conformal mapping. In Proc. 2nd Int. Conf. Numerical Methods in Fluid Dynamics (ed. M. Holt). Lecture Notes in Physics, vol 8, pages 458-462. Springer (Berlin Heidelberg).

Xue, S., Ko, J., and Xu, Y. (2000). Tuned liquid column damper for suppressing pitching motion of structures. Engineering Structures, 22(11), 1538-1551. 